\title{
CALCULATION OF THE MAXIMUM FORCE BETWEEN TWO COAXIAL CIRCULAR CURRENTS
}

\author{
By Frederick W. Grover
}

\section{CONTENTS}

I. Introduction

II. Formulas for the force between two coaxial circular coil

I. Maxwell's formula in zonal harmonics................ $3^{20}$

2. Maxwell's formula in elliptic integrals ................ $3^{2 I}$

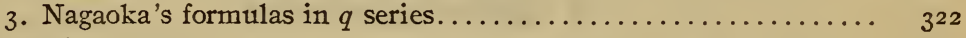

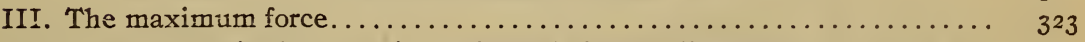

I. Rayleigh's approximate formula for the distance $z_{\mathrm{m}} \ldots \ldots \ldots \ldots, 3^{24}$

IV. Derivation of the new formulas........................... 325

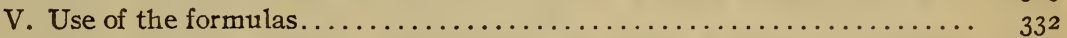

I. Differential formulas for calculating $z_{\mathrm{m}} \ldots \ldots \ldots \ldots \ldots \ldots \ldots \ldots, 33^{2}$

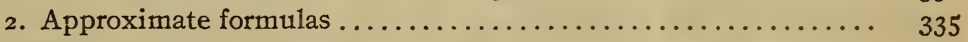

3. The calculation of the maximum force $\ldots \ldots \ldots \ldots \ldots \ldots \ldots .338$

VI. Variation formulas for the maximum force $F_{\mathrm{m}}$ and the maximum dis-

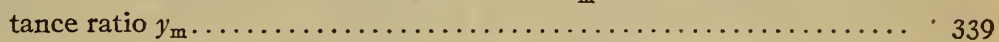

VII. Variation of the force for small displacements of the coils from the posi-

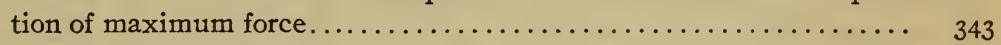

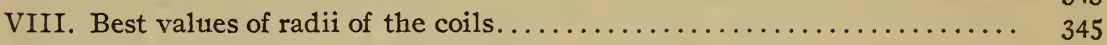

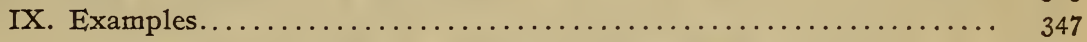

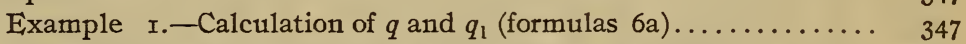

Example 2.-Illustrating the calculation of $y_{\mathrm{m}}$ by $(33) \ldots \ldots \ldots .349$

Example 3.-Calculation of $y_{m}$ by formula $(37) \ldots \ldots \ldots \ldots \ldots . \ldots \ldots$

Example 4.-Illustration of the use of the curve for obtaining a first approximation.................. $35^{2}$

Example 5.-Calculation by (37) from a first approximation derived from the curve.................. 353

Example 6.-Calculation of the factor $P \ldots \ldots \ldots \ldots \ldots \ldots \ldots \ldots, 354$

Example 7.-Calculation of the factor $X \ldots \ldots \ldots \ldots \ldots \ldots \ldots \ldots \ldots \ldots \ldots \ldots$

Example 8.-Use of differential formulas (38) and (39)........ 357

Example 9.-Calculation by differential formulas (40), (4I), and

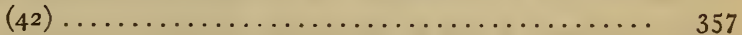

Example ro.-Calculation of the maximum force by formulas (34)

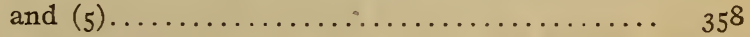

Example Ir.-Calculation of the maximum force by (37) and (6).. 359

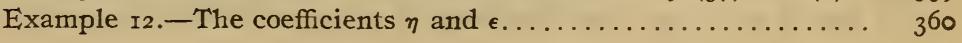


IX. Examples-Continued.

Page.

Example 13.-Illustrating calculation of maximum force for coils whose radii have a ratio differing slightly from one of the values in Table $4 \ldots \ldots \ldots \ldots \ldots \ldots \ldots, 3_{36} \mathrm{x}$

Example 14.-Calculation of the maximum force between the $\mathrm{Bu}$ reau of Standards current balance coils........ $\quad 3^{62}$

Table A.-Calculation of maximum force distance $z_{\mathrm{m}}$ for the Bureau

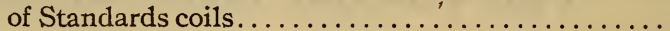

Table B.-Calculation of the maximum force for the Bureau of Standards coils............................

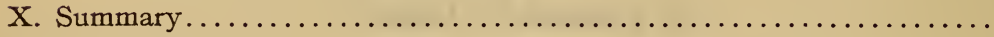

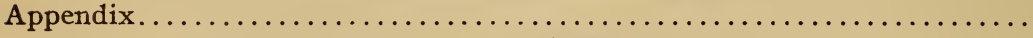

Table r. - Values of the Factor $P$ in Equation $\left(33^{2}\right) \ldots \ldots \ldots \ldots \ldots \ldots$

Table $2 .-$ Values of the Factor $X$ in Equation $(37 a) \ldots \ldots \ldots \ldots \ldots \ldots$

Table 3.-Values of the factors in the differential equations $\left(3^{8}\right)$ to $(42)$.

Table 4.-Fundamental values of the maximum force ratio $y_{m}$ and the

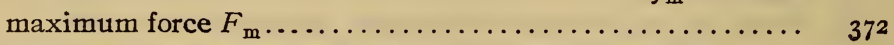

Table 5.-Coefficients $\eta$ and $\epsilon$ in Equations (48), (5I), (55), and (58)....

\section{INTRODUCTION}

Aside from its mathematical interest, the problem of the calculation of the force which exists between two circular currents has received a good deal of attention, owing to its practical importance in the calculation of the constants of apparatus used in absolute measurements. In such practical cases, it is true, the current is not concentrated in a circular filament, but flows in a number of wires, generally so disposed as to form a circular coil of rectangular cross section. The calculation of the mutual inductance of two such coils, as well as the force exerted between them, is, however, most readily based on the ideal case, a correction being applied to take into account the difference between the actual distribution of the current and the ideal distribution. This correction may be made with all the precision necessary, even in the most refined work, by the methods of Rayleigh and Lyle, provided that the dimensions of the cross sections are small with respect to the radii of the coils and the distance between them. In the formula of quadratures ${ }^{1}$ (Rayleigh) the coil is replaced by a number of circular filaments, the weighted mean being taken of their separate effects; in the method of Lyle ${ }^{2}$ the

${ }^{1}$ Gray, Absolute Meas., Vol. II, Part II, p. 403. This Bulletin, 2, p. 370; 1906 (Scientific Papers No. 42).

2 Phil. Mag., 3, p. 3ro; r902. Also, this Bulletin, 2, p. 374; 1906 (Scientific Papers No. 42). 
coil is shown to be equivalent to two circular filaments, whose radii and positions depend on the dimensions of the cross section. For details of such calculations the reader is referred to the original articles and to articles in this Bulletin. ${ }^{3}$

The mutual energy of two currents is equal to the product of their mutual inductance by the two current strengths. Accordingly, the force exerted by one circular filament on the other, in any specified direction, is equal to the rate of change of the mutual inductance as the filament moves in that direction multiplied by the product of the two current strengths. The force between two circular currents of unit strength may, therefore, be found by differentiating any suitable formula for their mutual inductance. The formulas of Maxwell and Nagaoka derived in this way for the special case of parallel coaxial circles are given for convenience of reference in the following section. These formulas allow of the calculation of the force with an accuracy which is limited only by the precision attainable in the measurement of the dimensions of the coils and the calculation of the correction for the finite cross section.

The present paper has to do with the special case, very important in practice, where the two parallel, coaxial, circular filaments are placed with such a distance between their planes that their mutual attraction or repulsion is a maximum. This is the condition which is realized in the form of current balance, first used by Lord Rayleigh ${ }^{4}$ in I884, and by Rosa, Dorsey, and Miller ${ }^{5}$ at the Bureau of Standards. In this form of balance a coil is suspended from one arm of a balance, with its plane midway between those of two horizontal fixed coils, the distances between the planes of the coils being so chosen that the forces exerted by the currents in the fixed coils on the current in the suspended coil are a maximum. This arrangement has the advantage that not only is the force sensibly constant for small displacements of the suspended coil from this ideal position, but the constant of the balance is a function of the ratio of the radii of the fixed and

${ }^{3}$ This Bulletin, 2, pp. 397-399, 1906 (Scientific Papers No. 42); and 8, p. 323, 331, 191 (Scientific Papers No. I69).

${ }^{4}$ Phil. Trans., 175, pp. 41 I-460, 1884; Rayleigh's Scientific Papers, 2, pp. 278-332.

5 This Bulletin, 8, pp. 269-393; I9II (Scientific Papers No. I69). 
movable coils, which ratio can be found with great accuracy by electrical methods, thus rendering unnecessary the measurement of the individual radii. The position of the coils for maximum force can be found by experiment.

The value of the maximum force can be calculated from the known ratio of the radii by interpolation between a number of values of the force, calculated by known formulas, for various distances in the neighborhood of the experimentally determined correct position of the coils. As far as is known to the author, this is the only method which has been previously used. The formulas and methods here developed are presented as affording a more direct solution of the problem, and although the amount of numerical work is, in the general case, still appreciable a check is afforded on the methods previously employed. For such cases as occur in practice, the constants may be obtained with very little labor from the tables of fundamental values given below.

The main formulas (33) to (37) were derived by the author in June, I9II. Since then, however, other work has interfered to delay the development of the auxiliary formulas and the preparation of the tables.

\section{FORMULAS FOR THE FORCE BETWEEN TWO COAXIAL CIRCULAR COILS.}

\section{MAXWELL'S FORMULA IN ZONAL HARMONICS. ${ }^{\circ}$}

Maxwell expressed the mutual inductance between two coaxial circular currents, whose radii $A$ and $a$ subtend, respectively, the angles $\alpha_{1}$ and $\alpha_{2}$ at the origin, and whose planes lie at distances $B$ and $b$ from this point, by a formula in zonal harmonics

$$
\begin{aligned}
M=4 \pi^{2} c \sin ^{2} \alpha_{1} \sin ^{2} \alpha_{2}\{ & \frac{1}{2} \frac{c}{C} P_{1}^{\prime}\left(\alpha_{1}\right) P_{1}^{\prime}\left(\alpha_{2}\right)+\cdots \\
& \left.+\frac{\mathrm{I}}{n(n+\mathrm{I})} \frac{c^{n}}{C^{n}} P_{n}^{\prime}\left(\alpha_{1}\right) P_{n}^{\prime}\left(\alpha_{2}\right)+\cdots \cdots\right\}
\end{aligned}
$$

where $c=\sqrt{a^{2}+b^{2}}$ and $C=\sqrt{A^{2}+B^{2}}$

Substituting for the zonal harmonics their well-known values in terms of sines and cosines, and introducing for the latter their 
values in terms of the radii and the distances $b, B$, and $c, C$, the formula (I) becomes

$$
\begin{aligned}
M & =\mathrm{I} .2 \pi^{2} \frac{A^{2} a^{2}}{C^{3}}+2.3 \pi^{2} \frac{A^{2} B a^{2} b}{C^{5}} \\
& +3.4 \pi^{2} \frac{A^{2} a^{2}\left(B^{2}-\frac{1}{4} A^{2}\right)\left(b^{2}-\frac{1}{4} a^{2}\right)}{C^{7}}+4.5 \pi^{2} \frac{B b\left(B^{2}-\frac{3}{4} A^{2}\right)\left(b^{2}-\frac{3}{4} a^{2}\right) A^{2} a^{2}}{C^{9}} \\
& +5.6 \pi^{2} \frac{\left(B^{4}-\frac{3}{2} B^{2} A^{2}+\frac{1}{8} A^{4}\right)\left(b^{4}-\frac{3}{2} b^{2} a^{2}+\frac{1}{8} a^{4}\right) A^{2} a^{2}}{C^{11}} \\
& +6.7 \pi^{2} \frac{B b\left(B^{4}-\frac{5}{2} B^{2} A^{2}+\frac{5}{8} A^{4}\right)\left(b^{4}-\frac{5}{2} b^{2} a^{2}+\frac{5}{8} a^{4}\right) A^{2} a^{2}}{C^{13}}+\ldots
\end{aligned}
$$

which on differentiation with respect to $b$ gives for the force 7

$$
\begin{gathered}
F=\frac{\pi^{2} A^{2} a^{2}}{C^{4}}\left\{1.2 .3 \frac{B}{C}+2.3 .4 \frac{b\left(B^{2}-\frac{1}{4} A^{2}\right)}{C^{3}}+3.4 .5 \frac{B\left(B^{2}-\frac{3}{4} A^{2}\right)\left(b^{2}-\frac{1}{4} a^{2}\right)}{C^{5}}\right. \\
+4.5 .6 \frac{B\left(B^{4}-\frac{3}{2} B^{2} A^{2}+\frac{1}{8} A^{4}\right)\left(b^{2}-\frac{3}{4} a^{2}\right) b}{C^{7}} \\
\left.+5.6 .7 \frac{B\left(B^{4}-\frac{5}{2} B^{2} A^{2}+\frac{5}{8} A^{4}\right)\left(b^{4}-\frac{3}{2} b^{2} a^{2}+\frac{1}{8} a^{4}\right)}{C^{9}}+\cdots\right\}
\end{gathered}
$$

On account of its slow convergence, the formula (3) is not suitable for numerical calculations. It is, however, valuable, in that it gives a clear view of the way the force varies when the distance between the coils is increased.

\section{MAXWELI'S FORMULA IN ELLIPTIC INTEGRALS. ${ }^{8}$}

An accurate expression for the force was derived by Maxwell by differentiating his well-known formula for the mutual inductance of two coaxial circles.

the result being

$$
M=-4 \pi \sqrt{A a}\left\{\left(k-\frac{2}{k}\right) K+\frac{2}{k} E\right\}
$$

$$
F=-\frac{\pi z \sin \gamma}{\sqrt{A a}}\left\{2 K-\left(\mathrm{I}+\sec ^{2} \gamma\right) E\right\}
$$

where $A$ and $a$ are the radii of the two circles, $z$ is the distance between their planes, and $K$ and $E$ are the complete elliptic integrals to modulus $k=\sin \gamma=\frac{2 \sqrt{A a}}{\sqrt{(A+a)^{2}+z^{2}}}$ 
A table was prepared by Lord Rayleigh giving the value of log $\left[\sin \gamma\left\{2 K-\left(\mathrm{I}+\sec ^{2} \gamma\right) E\right\}\right]$ to seven places of decimals. ${ }^{9}$ This table was recalculated at the Bureau of Standards, using Legendre's tables of elliptic integrals, the logarithms being carried out to ten places, and some small errors in the seventh place corrected. The table thus corrected was reproduced in the article on the absolute determination of the international ampere, by Rosa, Dorsey, and Miller.

\section{NAGAOKA'S FORMULAS}

The force between two parallel coaxial circles has also been expressed by Nagaoka ${ }^{10}$ in terms of the $q$ series of Jacobi. These expressions have the advantage of rapid convergence and of not requiring the use of tables of elliptic integrals. Nagaoka's formula (29), extended by the author of the present paper to include four additional terms, is

$$
\begin{aligned}
F=\frac{192 \pi^{2} z}{\sqrt{A a}} q^{\frac{s}{3}}[\mathrm{I} & +20 q^{2}+225 q^{4}+\mathrm{I} 840 q^{8} \\
& +\mathrm{I} 2120 q^{8}+6805^{2} q^{10} \\
& +337465 q^{12}+\mathrm{I} 5 \mathrm{I} 3740 q^{14} \\
& \left.+6247665 q^{16}+\ldots \ldots .\right]
\end{aligned}
$$

Extending Nagaoka's formula (2I), the author obtained the further formula which is equivalent to the formula (8) found by Nagaoka in a more recent article. ${ }^{11}$

$$
\begin{aligned}
& F=\frac{\pi z}{\mathrm{I} 6 q_{1} \sqrt{A a}}\left[\begin{array}{l}
\left(\mathrm{I}+\mathrm{I} 2 q_{1}-\mathrm{I} 92 q_{1}{ }^{2}+\mathrm{I} 232 q_{1}{ }^{3}-5634 q_{1}{ }^{4}\right. \\
\left.+2 \mathrm{I} 648 q_{1}{ }^{5}-73600 q_{1}{ }^{6}+226944 q_{1}{ }^{7}-648 \mathrm{I} 89 q_{1}{ }^{8}+\ldots\right)
\end{array}\right. \\
& \text { - I } 2 q_{1} \log _{\mathrm{e}} \frac{\mathrm{I}}{q_{1}} \cdot\left(\mathrm{I}-\mathrm{IO} q_{1}+60 q_{1}{ }^{2}-300 q_{1}{ }^{3}\right. \\
& +\mathrm{I} 300 q_{1}{ }^{4}-4884 q_{1}{ }^{5}+\mathrm{I} 6320 q_{1}{ }^{8} \\
& \left.\left.-49920 q_{1}{ }^{7}+142500 q_{1}^{8}-\cdots \cdots\right)\right]
\end{aligned}
$$

- Phil. Trans., 175, pp. 411-460, I884; Rayleigh's Scientific Papers, 2, 327.

${ }^{10}$ Jour. College of Science, Tokyo, vol. 16, art. 15, p. 12, 1903; Phil. Mag., 6, p. 19, 1903.

11 Tokyo Math.-Phys. Soc., vol. 6, p. I56, rgrr. 
where

$$
\begin{aligned}
& q=\frac{l}{2}+2\left(\frac{l}{2}\right)^{5}+\mathrm{I} 5\left(\frac{l}{2}\right)^{9}+\cdots \\
& \frac{l}{2}=\frac{\mathrm{I}}{2} \frac{\mathrm{I}-\sqrt{k^{\prime}}}{\mathrm{I}+\sqrt{\bar{k}^{\prime}}}=\frac{k^{2}}{2\left(\mathrm{I}+k^{\prime}\right)\left(\mathrm{I}+\sqrt{\left.\bar{k}^{\prime}\right)^{2}}\right.} \\
& k=\frac{2 \sqrt{A a}}{\sqrt{(A+a)^{2}+z^{2}}} \quad k^{\prime}=\sqrt{\mathrm{I}-k^{2}}=\frac{\sqrt{(A-a)^{2}+z^{2}}}{\sqrt{(A+a)^{2}+z^{2}}} \\
& q_{1}=\frac{l_{1}}{2}+2\left(\frac{l_{1}}{2}\right)^{5}+\mathrm{I} 5\left(\frac{l_{1}}{2}\right)^{9}+\cdots \ldots \\
& \frac{l_{1}}{2}=\frac{\mathrm{I}}{2} \frac{\mathrm{I}-\sqrt{k}}{\mathrm{I}+\sqrt{k}}=\frac{k^{\prime 2}}{2(\mathrm{I}+k)\left(\mathrm{I}+\sqrt{\bar{k})^{2}}\right.}
\end{aligned}
$$

In most cases only two or three terms need be calculated to give the force with a good deal of precision. The formulas are given at length here so as to give all needed precision, even in the most unfavorable cases. Formula (6) converges most rapidly for circles which are near together. It is not so convenient to use as (5) and will be employed only in those cases where the convergence of (5) is not satisfactory. With the number of terms included here, the force may be calculated by one or the other of the two formulas with an accuracy of better than I part in roo ooo, even in the most unfavorable case. To facilitate calculations, Nagaoka has published ${ }^{12}$ tables giving the values of $\frac{F \sqrt{A a}}{z}$ for different values of $q$.

Further formulas for the force have also been given by Nagaoka, for which the original articles already cited should be consulted.

\section{THE MAXIMUM FORCE}

The foregoing formulas suffice to give the value of the force between the circles with all the precision required in the most careful work.

For the calculation of the constant of the Rayleigh current balance we need, as has already been explained, to obtain either 
(a) an expression giving the maximum value of the force between the circular currents as a function of the ratio of their radii, or (b) a formula for calculating the value of the distance $z_{\mathrm{m}}$ which exists between their planes when the force is a maximum. The maximum force $F_{\mathrm{m}}$ may, in the latter case, be calculated by substituting $z_{\mathrm{m}}$ for $z$ in any one of the formulas' for the force given above, which converges satisfactorily.

Although more direct, the first method is, in some cases, less accurate than the second, and as a knowledge of the distance $z_{\mathrm{m}}$ is of interest in itself, formulas for both methods have been given below.

\section{RAYLEIGH'S APPROXIMATE FORMULA FOR $z_{m}$}

Obviously $z_{\mathrm{m}}$ may be found by differentiating any suitable expression for the force $F$ with respect to the distance $z$ between the circles, equating the result to zero, and solving for $z$. The result is the value $z_{\mathrm{m}}$ for which the force is a maximum. Provided the formula for $z_{\mathrm{m}}$ is not too complicated, it gives on substitution in the formula for the force an expression suitable for calculating the maximum force $F_{\mathrm{m}}$.

The differentiation of Maxwell's formula (3) gives, when the origin is taken at the center of the smaller circle $(b=0)$

$$
\begin{aligned}
& \left(\frac{d F}{d z}\right)_{b=0}=\frac{\pi^{2} A^{2} a^{2}}{C^{4}}\left[2 \cdot 3 \cdot 4 \frac{\left(z_{\mathrm{m}}{ }^{2}-\frac{1}{4} A^{2}\right)}{C^{3}}\right. \\
& \left.-3 \cdot 5 \cdot 6 \frac{a^{2}\left(z_{\mathrm{m}}{ }^{4}-\frac{3}{2} z_{\mathrm{m}}{ }^{2} A^{2}+\frac{1}{8} A^{4}\right)}{C^{7}}+\cdot \cdot\right]=0
\end{aligned}
$$

This may be solved for $z_{\mathrm{m}}$ by a method of approximation. The first term shows that $\frac{z_{\mathrm{m}}}{A}$ is less than unity. Taking as a first approximation that value of $\frac{z_{m}}{A}$ which makes the first term zero, and substituting this value in the second term, we find as a second approximation Rayleigh's equation ${ }^{13}$

or

$$
\frac{z_{\mathrm{m}}^{2}}{A^{2}}=\frac{\mathrm{I}}{4}\left(\mathrm{I}-\frac{9 a^{2}}{5 A^{2}}\right)
$$

$$
\frac{z_{\mathrm{m}}}{A}=\frac{\mathrm{I}}{2}\left(\mathrm{I}-\frac{9 a^{2}}{\mathrm{IO} A^{2}}-\cdots\right)
$$


It is not difficult, by substituting this value in the second and third terms of (7) to obtain a further approximation

$$
\frac{z_{\mathrm{m}}}{A}=\frac{\mathrm{I}}{2}\left(\mathrm{I}-\frac{9 a^{2}}{\mathrm{1O} A^{2}}-\frac{\mathrm{I}}{4} \frac{a^{4}}{A^{4}}\right)
$$

Unfortunately, the slow convergence of these expressions, and the rapid increase in the amount of labor necessary for obtaining each new term, prevent the use of (8) and (9) except for purposes of orientation, unless within the limited range where $\frac{a}{A}$ is small.

The differentiation of Maxwell's equation (4) in elliptic integrals is complicated and does not give a satisfactory formula for $z_{\mathrm{m}}$.

By following the method of Nagaoka the author of the present paper has obtained expressions in the form of $q$ series, which enable the values of $z_{\mathrm{m}}$ and $F_{\mathrm{m}}$ to be calculated with all the accuracy necessary in refined work, and these formulas have been tested thoroughly as to their applicability for numerical calculations and as to their agreement with the results found by other methods.

\section{DERIVATION OF THE NEW FORMULAS}

The mutual inductance of two parallel coaxial circles of radii $A$ and $a$, the distance between their planes being $z$, is given by the integral ${ }^{14}$

$$
M=4 \pi A a \int_{0}^{\pi} \frac{\cos \theta d \theta}{\sqrt{A^{2}+a^{2}+z^{2}-2 A a \cos \theta}}
$$

where $\theta$ is the angle between two elements $d s$ and $d s^{\prime}$ of the two circles.

Differentiating this with respect to $z$, we find for the force

$$
F=\frac{d M}{d z}=4 \pi A a z \int_{0}^{\pi} \frac{\cos \theta d \theta}{\left(A^{2}+a^{2}+z^{2}-2 A a \cos \theta\right)^{\frac{3}{2}}}
$$

and the condition which must hold when the force between the circles is a maximum is

$$
\begin{aligned}
\frac{d F}{d z}=\frac{d^{2} M}{d z^{2}} & =4 \pi A a \int_{0}^{\pi} \frac{\cos \theta d \theta}{\left(A^{2}+a^{2}+z^{2}-2 A a \cos \theta\right)^{\frac{3}{2}}} \\
& +4 \pi A a z^{2} \int_{0}^{\pi} \frac{3 \cos \theta d \theta}{\left(A^{2}+a^{2}+z^{2}-2 A a \cos \theta\right)^{\frac{5}{2}}}=0
\end{aligned}
$$


Nagaoka in the first of the papers above cited has shown how to express the elliptic integral (I I) in elliptic functions, and by expanding these in terms of $q$ functions has obtained the formulas (5) and (6) (the latter in a different form) for the force, which have been given above.

For the integration of (12) we need evaluate only one new integral-the second. For completeness, however, we will also indicate the solution of the first integral as given by Nagaoka.

Put

then

$$
\cos \theta=\alpha s+\beta
$$

$$
\begin{gathered}
-\sin \theta d \theta=\alpha d s \\
\sin ^{2} \theta=\mathrm{I}-(\alpha s+\beta)^{2}=-\alpha^{2} s^{2}-2 \alpha \beta s+\left(\mathrm{I}-\beta^{2}\right) \\
A^{2}+a^{2}+z^{2}-2 A a \cos \theta=\left[\left(a^{2}+A^{2}+z^{2}-2 a A \beta\right)-2 a A \alpha s\right]
\end{gathered}
$$

and therefore,

$$
\begin{aligned}
& \sin \theta \sqrt{A^{2}+a^{2}+z^{2}-2 A a \cos \theta}= \\
& \quad \sqrt{\left[A^{2}+a^{2}+z^{2}-2 a A \beta-2 a A \alpha s\right]\left[-\alpha^{2} s^{2}-2 \alpha \beta s+\left(\mathrm{I}-\beta^{2}\right)\right]}
\end{aligned}
$$

In order that the cubic under the radical may assume the canonical Weierstrassian form

$$
\sqrt{4\left(s-e_{1}\right)\left(s-e_{2}\right)\left(s-e_{3}\right)}=\sqrt{4 s^{3}-g_{2} s-g_{3}}=\sqrt{S}
$$

the following conditions must be satisfied

which give

$$
\begin{aligned}
2 a A \alpha^{3} & =4 \\
e_{1}+e_{2}+e_{3} & =6 a A \beta \alpha^{2}-\alpha^{2}\left(a^{2}+A^{2}+z^{2}\right)=0
\end{aligned}
$$

$$
\alpha=\left(\frac{2}{A a}\right)^{\frac{1}{3}}, \quad \beta=\frac{A^{2}+a^{2}+z^{2}}{6 A a}
$$

$A^{2}+a^{2}+z^{2}-2 A a \cos \theta=\frac{4}{\alpha^{3}}(2 \beta-\alpha s)$

whence

$$
\frac{\mathrm{I}}{4} g_{2}=\frac{3 \beta^{2}+\mathrm{I}}{\alpha^{2}} \quad \frac{\mathrm{I}}{4} g_{3}=\frac{2 \beta\left(\beta^{2}-\mathrm{I}\right)}{\alpha^{3}}
$$

$$
e_{1}=\frac{2 \beta}{\alpha} \quad e_{2}=\frac{I-\beta}{\alpha} \quad e_{3}=-\frac{(I+\beta)}{\alpha}
$$


We have accordingly for the integrand of the first integral

$$
\left(\frac{\cos \theta d \theta}{\left(A^{2}+a^{2}+z^{2}-2 A a \cos \theta\right)^{\frac{3}{2}}}=-\frac{\alpha d s(\alpha s+\beta)}{\sqrt{S} \cdot \frac{4}{\alpha^{3}}(2 \beta-\alpha s)}\right.
$$

and for the limits we notice that, when

$$
\begin{aligned}
& \theta=\pi, \alpha s+\beta=-\mathrm{I} \text { and therefore } s=e_{3} \\
& \theta=0, \alpha s+\beta=\mathrm{I} \text { and therefore } s=e_{2}
\end{aligned}
$$

Introducing these conditions, the first integral becomes

$$
I_{1}=4 \pi A a \int_{e_{2}}^{e_{3}}\left[-\frac{\alpha^{4}}{4} \frac{(\alpha s+\beta)}{(2 \beta-\alpha s)} \cdot \frac{d s}{\sqrt{S}}\right]
$$

Introducing the Weierstrassian function, $\mathrm{p} u$ which is defined by the equations $u=\int_{\mathrm{s}}^{\infty} \frac{d s}{\sqrt{S}}$ and $s=\mathrm{p} u$

and remembering that when $s=e_{3}, u=\omega_{3}$; and when $s=e_{2}, u=\omega_{2}$, the integral becomes

$$
I_{1}=-4 \pi A a \int_{\omega_{3}}^{\omega_{2}} \frac{\alpha^{4}}{4} \frac{(\alpha \mathrm{p} u+\beta)}{(\alpha \mathrm{p} u-2 \beta)} d u
$$

The integrand may be written

$$
\mathrm{I}+\frac{3 \beta}{\alpha \mathrm{p} u-2 \beta}=\mathrm{I}+\frac{3}{2} \frac{e_{1}}{\mathrm{p} u-e_{1}}
$$

which, taking into account the relations

$$
\begin{aligned}
\frac{\mathrm{I}}{\mathrm{p} u-e_{1}} & =-\frac{e_{1}}{\left(e_{1}-e_{2}\right)\left(e_{1}-e_{3}\right)}+\frac{\mathrm{p}\left(u \pm \omega_{1}\right)}{\left(e_{1}-e_{2}\right)\left(e_{1}-e_{3}\right)} \\
\omega_{2}-\omega_{3} & =\omega_{1} \\
\int_{\omega_{3}}^{\omega_{3}} \mathrm{p}\left(u \pm \omega_{1}\right) d u & =-\eta_{1}
\end{aligned}
$$

gives finally

$$
\frac{I_{1}}{4 \pi A a}=-\frac{\alpha^{4}}{4}\left[\omega_{1}-\frac{3}{2} \frac{e_{1}}{\left(e_{1}-e_{2}\right)\left(e_{1}-e_{3}\right)}\left(\eta_{1}+e_{1} \omega_{1}\right)\right]
$$


From what has gone before, the second integral $I_{2}$ can be shown to be equivalent to

$$
I_{2}=\frac{\alpha^{7}}{\mathrm{I} 6} \int_{\omega_{3}}^{\omega_{2}} \frac{[\alpha \mathrm{p} u+\beta]}{[\alpha \mathrm{p} u-2 \beta]^{2}} d u
$$

The integrand may be written

$$
\frac{\mathrm{I}}{\alpha} \frac{\mathrm{I}}{\mathrm{p} u-e_{1}}+\frac{3}{2} \frac{e_{1}}{\alpha} \frac{\mathrm{I}}{\left(\mathrm{p} u-e_{1}\right)^{2}}
$$

and the first term can be integrated by (22). For the second term we make use of the relations

$$
\begin{gathered}
\frac{\left(e_{1}-e_{2}\right)^{2}\left(e_{1}-e_{3}\right)^{2}}{\left(\mathbf{p} u-e_{1}\right)^{2}}=\mathbf{p}^{2}\left(u \pm \omega_{1}\right)-2 e_{1} \mathbf{p}\left(u \pm \omega_{1}\right)+e_{1}{ }^{2} \\
\mathbf{p}^{2}\left(u+\omega_{1}\right)=\frac{1}{6} \mathbf{p}^{\prime \prime} u+\frac{g_{2}}{\mathrm{I} 2}
\end{gathered}
$$

which, remembering that $\mathrm{p}^{\prime} \omega_{3}=0$, and $\mathrm{p}^{\prime} \omega_{2}=0$ gives, finally, for the second term

$$
\left(e_{1}-e_{2}\right)^{2}\left(e_{1}-e_{3}\right)^{2} \int_{\omega_{3}}^{\omega_{2}} \frac{d u}{\left(\mathbf{p} u-e_{1}\right)^{2}}=\frac{y_{2}}{12} \omega_{1}+2 e_{1} \eta_{1}+e_{1}^{2} \omega_{1}
$$

so that the integral $I_{2}$ becomes

$$
I_{2}=\frac{\alpha^{6}}{\mathrm{I} 6}\left[-\frac{\left(\eta_{1}+e_{1} \omega_{1}\right)}{\left(e_{1}-e_{2}\right)\left(e_{1}-e_{3}\right)}+\frac{3}{2} \frac{e_{1}}{\left(e_{1}-e_{2}\right)^{2}\left(e_{1}-e_{3}\right)^{2}}\left(\frac{g_{2}}{12} \omega_{1}+2 e_{1} \eta_{1}+e_{1}^{2} \omega_{1}\right)\right]
$$

The integrated form of equation (12) is therefore found to be

$$
\begin{gathered}
\frac{\mathrm{I}}{4 \pi A a} \frac{\partial^{2} M}{\partial z^{2}}=-\frac{\alpha^{4}}{4}\left[\omega_{1}-\frac{3}{2} \frac{e_{1}}{\left(e_{1}-e_{2}\right)\left(e_{1}-e_{3}\right)}\left(\eta_{1}+e_{1} \omega_{1}\right)\right] \\
-\frac{3}{\mathrm{I} 6} \alpha^{6} z^{2}\left[-\frac{\left(\eta_{1}+e_{1} \omega_{1}\right)}{\left(e_{1}-e_{2}\right)\left(e_{1}-e_{3}\right)}+\frac{3}{2} \frac{e_{1}}{\left(e_{1}-e_{2}\right)^{2}\left(e_{1}-e_{3}\right)^{2}}\left(e_{1}{ }^{2} \omega_{1}+2 e_{1} \eta_{1}+\frac{g_{2}}{\mathrm{I} 2} \omega_{1}\right)\right]
\end{gathered}
$$

For numerical calculations we have next to express the formula just derived in terms of the $\theta$ functions of Jacoby, from which the transition to the $q$ series may readily be made. 
It is convenient to write the last term of (28) in the form

$$
\frac{3}{2} \frac{e_{1}{ }^{2}}{\left(e_{1}-e_{2}\right)^{2}\left(e_{1}-e_{3}\right)^{2}}\left[\left(\eta_{1}+e_{1} \omega_{1}\right)+\left(\eta_{1}+\frac{g_{2}}{12} \frac{\omega_{1}}{e_{1}}\right)\right]
$$

and then, using the relations,

$$
\begin{aligned}
& \eta_{1}+e_{1} \omega_{1}=-\frac{\mathrm{I}}{4 \omega_{1}} \frac{\theta_{2}{ }^{\prime \prime}(\mathrm{o})}{\theta_{2}(\mathrm{o})}, e_{1}-e_{3}=\frac{\pi^{2}}{4 \omega_{1}{ }^{2}} \theta_{3}{ }^{4}(\mathrm{o}), e_{1}-e_{2}=\frac{\pi^{2}}{4 \omega_{1}{ }^{2}} \theta_{0}{ }^{4}(\mathrm{o}) \\
& \left.e_{1}=\frac{\mathrm{I}}{3} \frac{\pi^{2}}{4 \omega_{1}{ }^{2}}\left[\theta_{3}{ }^{4}(\mathrm{o})+\theta_{0}{ }^{4}(\mathrm{o})\right], \frac{g_{2}}{\mathrm{I} 2}=\frac{\mathrm{I}}{\mathrm{I} 8} \frac{\pi^{4}}{\mathrm{I} 6 \omega_{1}{ }^{4}}\left[\theta_{0}{ }^{8}(\mathrm{o})+\theta_{2}{ }^{8}(\mathrm{o})+\theta_{3}{ }^{8}(\mathrm{o})\right]\right\} \\
& \eta_{1} \omega_{1}=-\frac{\mathrm{I} \theta_{1}^{\prime \prime \prime}(\mathrm{o})}{\mathrm{I} 2 \theta_{1}^{\prime}(\mathrm{o})}=-\frac{\mathrm{I}}{\mathrm{I} 2}\left[\frac{\theta_{0}^{\prime \prime}(\mathrm{o})}{\theta_{0}(\mathrm{o})}+\frac{\theta_{2}^{\prime \prime}(\mathrm{o})}{\theta_{2}(\mathrm{o})}+\frac{\theta_{3}^{\prime \prime}(\mathrm{o})}{\theta_{3}(\mathrm{o})}\right]
\end{aligned}
$$

we find, finally, that the condition which must be satisfied by $z_{m}$ becomes

$$
\begin{aligned}
\frac{3}{\mathrm{I} 6} \alpha^{6} z_{m^{2}}{ }^{2} & {\left[\frac{4}{\pi^{4}} \frac{\theta_{2}^{\prime \prime}}{\theta_{2}} \frac{\mathrm{I}}{\left(\theta_{3}^{4}+\theta_{0}^{4}\right)}-\frac{2}{9 \pi^{4}}\left(\frac{\mathrm{I}}{\theta_{0}^{4}}+\frac{\mathrm{I}}{\theta_{3}^{4}}\right)\left(\frac{\theta_{0}^{\prime \prime}}{\theta_{0}}+4 \frac{\theta_{2}^{\prime \prime}}{\theta_{2}}+\frac{\theta_{3}^{\prime \prime}}{\theta_{3}}\right)\right.} \\
& \left.\frac{-2}{9 \pi^{2}}\left(\mathrm{I}-\frac{\theta_{0}^{4}}{\theta_{3}^{4}}-\frac{\theta_{3}^{4}}{\theta_{0}^{4}}\right)\right]=-\frac{\alpha^{4}}{\omega_{1}^{2}}\left[\frac{\theta_{3}^{4} \theta_{0}^{4}}{\left(\theta_{0}^{4}+\theta_{3}^{4}\right)}+\frac{\mathrm{I}}{2 \pi^{2}} \frac{\theta_{2}^{\prime \prime}}{\theta_{2}}\right]
\end{aligned}
$$

all the $\theta$ functions being to argument zero.

To find an expression involving $q$ functions we make use of the well known relations

$$
\begin{aligned}
& \theta_{0}=\mathrm{I}-2 q+2 q^{4}-2 q^{9}+\ldots \ldots+(-\mathrm{I})^{\mathrm{n}} 2 q^{\mathrm{n}^{2}}+\ldots . \\
& \theta_{3}=\mathrm{I}+2 q+2 q^{4}+2 q^{9}+\ldots \ldots+2 q^{\mathrm{n}^{2}}+\ldots \ldots \\
& \theta_{2}=2 q^{\frac{1}{4}}\left[\mathrm{I}+q^{2}+q^{6}+q^{12}+\ldots \ldots+q^{\mathrm{n}(\mathrm{n}+1)}+\ldots . .\right]
\end{aligned}
$$

The substitution of these values gives rise to long and tedious reductions and only the final result will be given. This is

$$
\begin{aligned}
& z_{\mathrm{m}}{ }^{2}=\frac{A a}{20 q}\left[\mathrm{I}-36 q^{2}+722 q^{4}-\mathrm{I} \mathrm{I} 208 q^{8}+\mathrm{I} 55487 q^{8}\right. \\
& -2065492 q^{10}+27054638 q^{12} \\
& \left.-353044772 q^{14}+4603353515 q^{16}-. . \cdot \cdot\right]
\end{aligned}
$$

or, if the square root be taken,

$$
\left.\begin{array}{rl}
z_{\mathrm{m}}=\frac{\sqrt{A a}}{2 \sqrt{5 q}}[\mathrm{I} & -\mathrm{I} 8 q^{2}+\mathrm{I} 99 q^{4}-2022 q^{6}+21547 q^{8} \\
& -24222 q^{10}+2829828 q^{12} \\
& -33755570 q^{14}+408424637 q^{16} \ldots . .
\end{array}\right]
$$

Substituting this value of $z_{\mathrm{m}}$ in equation (5) we derive for the maximum force (putting for $q$ the value $q_{0}$ corresponding to $z_{\mathrm{m}}$ )

$$
\begin{aligned}
& \begin{array}{c}
F_{\mathrm{m}}=\frac{96}{\sqrt{5}} \pi^{2} q_{0}{ }^{2}\left[\mathrm{I}+2 q_{0}{ }^{2}+64 q_{0}{ }^{4}-252 q_{0}{ }^{6}+4882 q_{0}{ }^{8}\right. \\
-50480 q_{0}{ }^{10}+63 \mathrm{I} 392 q_{0}{ }^{12}
\end{array} \\
& \left.-7604902 q_{0}{ }^{14}+93488433 q^{18}-\ldots \cdot\right] \\
& 18423^{\circ}-16-2
\end{aligned}
$$


In all the equations thus far given the elliptic functions have had as semiperiods the quantities $\omega_{1}$ and $\omega_{3}$, respectively real and pure imaginary, and $q=e^{i \pi \tau}$, where $\tau=\frac{\omega_{3}}{\omega_{1}}$ and $i=\sqrt{-\mathrm{I}}$. It can, however, be shown that a given expression can be made to depend also on elliptic functions having for periods $\omega_{3}$ and $-\omega_{1}$, respectively real and imaginary, if attention be paid to the following points. The invariants $g_{2}$ and $g_{3}$, and the quantity $e_{2}$ are unchanged by the transformation. The remaining quantities $e_{1}, e_{3}, k, k^{\prime}, \tau, q=e^{i \pi \tau}$ go into the following, respectively, $e_{3}, e_{1}, k^{\prime}$, $k, \tau_{1}=-\frac{\mathrm{I}}{\tau}, q^{\prime}=e^{i \pi \tau_{1}}$.

We may, consequently, write (28) in the form

$$
\begin{aligned}
\frac{\mathrm{I}}{4 \pi A a} \frac{\partial^{2} M}{\partial z^{2}}= & -\frac{\alpha^{4}}{4} \frac{\mathrm{I}}{\omega_{3}}\left[\omega_{1} \omega_{3}-\frac{3 e_{1}}{2\left(e_{1}-e_{2}\right)\left(e_{1}-e_{3}\right)}\left(\eta_{1} \omega_{3}+e_{1} \omega_{1} \omega_{3}\right)\right] \\
& -\frac{3 \alpha^{6}}{\mathrm{I} 6} z_{\mathrm{m}}{ }^{2}\left[-\frac{\mathrm{I}}{\omega_{3}}\left(\eta_{1} \omega_{3}+e_{1} \omega_{1} \omega_{3}\right)\right. \\
& \left.+\frac{3}{2} \frac{e_{1}{ }^{2}}{\left(e_{1}-e_{2}\right)^{2}\left(e_{1}-e_{3}\right)^{2}} \frac{\mathrm{I}}{\omega_{3}}\left\{2 \eta_{1} \omega_{3}+e_{1} \omega_{1} \omega_{3}+\frac{g_{2}}{\mathrm{I} 2} \frac{\omega_{1} \omega_{3}}{e_{1}}\right\}\right]
\end{aligned}
$$

and for the transformation to $\theta$ functions introduce into this the relations

$$
\begin{aligned}
& \eta_{1} \omega_{3}=\omega_{1} \eta_{3}+i \frac{\pi}{2}, \quad \frac{\omega_{1}}{\omega_{3}}=\frac{\mathrm{I}}{\pi i} \log _{\mathrm{e}} \frac{\mathrm{I}}{q_{1}} \\
& \eta_{1} \omega_{3}+e_{1} \omega_{1} \omega_{3}=\frac{\omega_{1}}{\omega_{3}}\left(\omega_{3} \eta_{3}+e_{1} \omega_{3}{ }^{2}\right)+\frac{\pi i}{2} \\
& e_{1} \omega_{3}{ }^{2}+\omega_{3} \eta_{3}=-\frac{\mathrm{I}}{4} \frac{\theta_{0}^{\prime \prime}\left(\mathrm{o} / \tau_{1}\right)}{\theta_{\mathrm{o}}\left(\mathrm{o} / \tau_{1}\right)} \\
& e_{1}-e_{2}=-\frac{\pi^{2}}{4 \omega_{3}{ }^{2}} \theta_{2}{ }^{4}\left(\mathrm{o} / \tau_{1}\right), \quad e_{1}-e_{3}=-\frac{\pi^{2}}{4 \omega_{3}{ }^{2}} \theta_{0}{ }^{4}\left(\mathrm{o} / \tau_{1}\right) \\
& e_{1}=-\frac{1}{3} \frac{\pi^{2}}{4 \omega_{3}{ }^{2}}\left[\theta_{2}{ }^{4}\left(\mathrm{o} / \tau_{1}\right)+\theta_{3}{ }^{4}\left(\mathrm{o} / \tau_{1}\right)\right] \\
& g_{2}=\frac{2}{3}\left(\frac{\pi^{2}}{2 \omega_{3}}\right)^{4}\left[\theta_{0}{ }^{8}\left(\mathrm{o} / \tau_{1}\right)+\theta_{2}{ }^{8}\left(\mathrm{o} / \tau_{1}\right)+\theta_{3}{ }^{8}\left(\mathrm{o} / \tau_{1}\right)\right]
\end{aligned}
$$


which give

$$
\begin{aligned}
& \frac{3 z_{\mathrm{m}}^{2}}{\mathrm{I} 6 A^{2} a^{2}}\left[-\log _{\mathrm{e}} \frac{\mathrm{I}}{q_{1}}\left\{\frac{4}{\pi^{4}} \frac{\theta_{0}^{\prime \prime}}{\theta_{0}} \frac{\mathrm{I}}{\theta_{2}{ }^{4} \theta_{3}{ }^{4}}-\frac{2}{9}\left(\frac{\mathrm{I}}{\theta_{3}{ }^{4}}+\frac{\mathrm{I}}{\theta_{2}{ }^{4}}\right)^{2}\left(\frac{\theta_{2}{ }^{\prime \prime}}{\theta_{2}}+\frac{\theta_{3}{ }^{\prime \prime}}{\theta_{3}}+4 \frac{\theta_{0}^{\prime \prime}}{\theta_{0}}\right)\right.\right. \\
& \left.+\frac{2}{9 \pi^{2}}\left(\frac{\mathrm{I}}{\theta_{3}^{4}}+\frac{\mathrm{I}}{\theta_{2}^{4}}\right)\left(\mathrm{I}-\frac{\theta_{2}^{4}}{\theta_{3}^{4}}-\frac{\theta_{3}^{4}}{\theta_{2}^{4}}\right)\right\} \\
& \left.-\frac{8}{\pi^{2}} \frac{\mathrm{I}}{\theta_{3}{ }^{4} \theta_{2}{ }^{4}}+\frac{8}{3 \pi^{2}}\left(\frac{\mathrm{I}}{\theta_{3}{ }^{4}}+\frac{\mathrm{I}}{\theta_{2}{ }^{4}}\right)^{2}\right] \\
& =\frac{\mathrm{I}}{\pi^{2} A a \theta_{0}^{4}}\left[\left(\frac{\mathrm{I}}{\theta_{3}^{4}}+\frac{\mathrm{I}}{\theta_{2}^{4}}\right)-\log _{\ominus} \frac{\mathrm{I}}{q_{1}}\left(\mathrm{I}-\frac{\mathrm{I}}{2 \pi^{2}} \frac{\theta_{0}^{\prime \prime}}{\theta_{0}}\left(\frac{\mathrm{I}}{\theta_{3}^{4}}+\frac{\mathrm{I}}{\theta_{2}^{4}}\right)\right]\right.
\end{aligned}
$$

and on substituting for these $\theta$ functions, whose argument is zero, and whose periods have the ratio $\tau_{1}$, expansions exactly like those of (3I) excepting that $q_{1}$ appears in place of $q$, we obtain a second expression for the distance $z_{\mathrm{m}}$ corresponding to the maximum force.

$$
\begin{aligned}
& z_{\mathrm{m}}{ }^{2}\left[\left(\mathrm{I}-\mathrm{I} 6 q_{1}+376 q_{1}{ }^{2}-4672 q_{1}{ }^{3}+38948 q_{1}{ }^{4}-252192 q_{1}{ }^{5}\right.\right. \\
& \left.+\mathrm{I} 365888 q_{1}{ }^{6}-6463360 q_{1}{ }^{7}+27500946 q_{1}{ }^{8}-\ldots . .\right) \\
& -\mathrm{I} 20 q_{1}{ }^{2} \log _{\mathrm{e}} \frac{\mathrm{I}}{q_{1}} \cdot\left(\mathrm{I}-\mathrm{I} 6 q_{1}+\mathrm{I} 54 q_{1}{ }^{2}-\mathrm{I} \mathrm{I} 2 \mathrm{OO} q_{1}{ }^{3}+6680 q_{1}{ }^{4}\right. \\
& \left.\left.-34272 q_{1}{ }^{5}+\mathrm{I}_{5} 6268 q_{1}{ }^{6}-\ldots .\right)\right] \\
& =32 a A q_{1}\left[\left(\mathrm{I}+24 q_{1}+36 q_{1}{ }^{2}+384 q_{1}{ }^{3}+402 q_{1}{ }^{4}+3456 q_{1}{ }^{5}\right.\right. \\
& \left.+3064 q_{1}{ }^{6}+23040 q_{1}{ }^{7}+18351 q_{1}^{8}+\ldots .\right) \\
& -\mathrm{I} 2 q_{1} \log _{e} \frac{\mathrm{I}}{q_{1}} \cdot\left(\mathrm{I}+2 q_{1}+24 q_{1}{ }^{2}+28 q_{1}{ }^{3}+264 q_{1}{ }^{4}\right. \\
& \left.\left.+25^{2} q_{1}{ }^{5}+2016 q_{1}{ }^{6}+1696 q_{1}{ }^{7}+12264 q_{1}{ }^{8}+\ldots .\right)\right]
\end{aligned}
$$

It does not seem advisable to substitute this equation in (6) to obtain an expression for the maximum force $F_{m}$, but, rather, to calculate numerical values of $z_{\mathrm{m}}$ from (37) and to substitute this numerical value of $z_{\mathrm{m}}$ in (6). 


\section{USE OF THE FORMULAS}

It is convenient to write the two formulas (33) and (37) just derived in the abbreviated forms

and

$$
y_{\mathrm{m}}=\frac{z_{\mathrm{m}}}{A}=\sqrt{\frac{a}{A} \cdot \frac{\mathrm{I}}{2 \mathrm{O} q}} \cdot P=\sqrt{\frac{\alpha}{2 \mathrm{O} q}} \cdot P
$$

$$
\left(\frac{z_{\mathrm{m}}}{A}\right)^{2}\left[S-\mathrm{I} 20 q_{1}^{2} \log _{\mathrm{e}} \frac{\mathrm{I}}{q_{1}} \cdot T\right]=32 \frac{a}{A} q_{1}\left[Q-\mathrm{r} 2 q_{1} \log _{\mathrm{e}} \frac{\mathrm{I}}{q_{1}} \cdot R\right]
$$

or

$$
y_{\mathrm{m}}{ }^{2}=\left(\frac{z_{\mathrm{m}}}{A}\right)^{2}=32 \frac{a}{A} q_{1} X=32 \alpha q_{1} X
$$

respectively. Tables of values of the quantities $P$ and $X$ have been calculated to aid in using these formulas, and will be found in the appendix. Examples illustrating the use of these tables are given below.

In employing the formulas just derived, we are met by the difficulty that the quantity $z_{\mathrm{m}}$ depends on either $q$ or $q_{1}$, which, in their turn, depend on the moduli $k^{\prime}$ and $k$, and the latter, as (6a) shows us, involve the very quantity $z_{\mathrm{m}}$ which we wish to calculate. Since, however, the variations of $q$ and $q_{1}$ with small changes of $z_{\mathrm{m}}$ are not important, we may solve our equations by successive approximation, and fortunately, if we know at the start, the approximate value of $z_{\mathrm{m}}$, it is not a difficult matter to obtain the true value with a great deal of precision, after only a few trials. This process may be expedited by the use of suitable differential formulas, or by use of the tables of values of $P$ and $X$.

\section{DIFFERENTIAL FORMULAS FOR CALCULATING $z_{m}$}

For small changes in $z$ due to a given change in $q$, we have, in the neighborhood of $\left(z=z_{\mathrm{m}}\right)$, with $\alpha$ constant,

$$
\frac{\Delta y}{y}=\frac{\Delta z}{z}=\frac{\mathrm{I}}{2} \frac{\Delta q}{q}\left(\mathrm{I}+72 q^{2}-296 q^{4}+4608 q^{6}-57320 q^{8}+\cdots \cdot\right)
$$

as can be verified by differentiating (32).

Similarly, by differentiating (6a), and putting for $q$ its approximate value $\frac{l}{2}$, we find the general expression ( $\alpha$ constant) 


$$
\begin{aligned}
\frac{\Delta q}{q}= & -\frac{\Delta z \quad k^{4}\left(\mathrm{I}+\mathrm{IO} q^{4}\right)}{z \frac{a}{A} k^{\prime \frac{3}{2}}\left(\mathrm{I}-k^{\prime}\right)} \cdot \frac{z^{2}}{A^{2}}=-\frac{\Delta z}{z} \cdot \frac{k^{2}\left(\mathrm{I}+k^{\prime}\right)}{4 \frac{a}{A} k^{\prime \frac{1}{3}}} \cdot \frac{z^{2}}{A^{2}} \cdot\left(\mathrm{I}+\mathrm{IO} q^{4}\right) \\
& =-\frac{\Delta y}{y} \cdot \frac{k^{2}\left(\mathrm{I}+k^{\prime}\right)}{4 \alpha k^{\prime \frac{3}{2}}} y^{2}\left(\mathrm{I}+\mathrm{IO} q^{4}\right)
\end{aligned}
$$

Differentiating (37) we may also find an expression corresponding to (38). The result is not, however, simple. Writing (37) in the form $z_{\mathrm{m}}{ }^{2} C_{1}=32 A a q_{1} C_{2}$, we find (for constant $\alpha$ )

$$
\begin{aligned}
& \frac{\Delta y}{y}=\frac{\Delta z}{z}=\frac{\mathrm{I}}{2} \frac{\Delta q_{1}}{q_{1}}\left(\mathrm{I}-\frac{q_{1}}{C_{1}} \frac{d C_{1}}{d q_{1}}+\frac{q_{1}}{C_{2}} \frac{d C_{2}}{d q_{1}}\right) \\
& \frac{d C_{1}}{d q_{1}}=-\mathrm{I} 6\left[\mathrm{I}-\frac{\mathrm{IO} 9}{2} q_{1}+996 q_{1}{ }^{2}-\operatorname{I08} 92 q_{1}{ }^{3}+87210 q_{1}{ }^{4}\right. \\
& \left.-562308 q_{1}{ }^{5}+\cdots \cdot\right] \\
& -240 q_{1} \log _{\mathrm{e}} \frac{\mathrm{I}}{q_{1}}\left[\mathrm{I}-24 q_{1}+308 q_{1}{ }^{2}-2800 q_{1}{ }^{3}+20040 q_{1}{ }^{4}\right. \\
& \text { - II } \left.9952 q_{1}{ }^{5}+625072 q_{1}{ }^{6}-\cdots \cdot\right] \\
& \frac{d C_{2}}{d q_{1}}=36\left[\mathrm{I}+\frac{8}{3} q_{1}+40 q_{1}{ }^{2}+54 q_{1}{ }^{3}+568 q_{1}{ }^{4}+\frac{\mathrm{I} 784}{3} q_{1}{ }^{5}\right. \\
& \left.+5^{\mathrm{I}} 5^{2} q_{1}{ }^{6}+\frac{\mathrm{I} 3930}{3} q_{1}{ }^{7}+\cdots \cdot\right] \\
& \text {-I } 2 \log _{\mathrm{e}} \frac{\mathrm{I}}{q_{1}}\left[\mathrm{I}+4 q_{1}+72 q_{1}{ }^{2}+\mathrm{II} 3 q_{1}{ }^{3}+\mathrm{I} 320 q_{1}{ }^{4}+\mathrm{I} 5 \mathrm{I} 2 q_{1}{ }^{5}\right. \\
& \text { + I } \left.4 \text { I I } 2 q_{1}{ }^{6}+\text { I } 3568 q_{1}{ }^{7}+\text { I } 10376 q_{1}{ }^{8}+\cdots \cdot\right] \text { ] } \\
& C_{1}=\left[\mathrm{I}-\mathrm{I} 6 q_{1}+376 q_{1}{ }^{2}-4672 q_{1}{ }^{3}+38948 q_{1}{ }^{4}-252192 q_{1}{ }^{5}\right. \\
& \left.+\mathrm{r} 365888 q_{1}{ }^{6}-\cdots \cdot\right] \\
& \text { - I } 20 q_{1}^{2} \log _{\mathrm{e}} \frac{\mathrm{I}}{q_{1}}\left[\mathrm{I}-\mathrm{I} 6 q_{1}+\mathrm{I} 54 q_{1}{ }^{2}-\mathrm{I} \mathrm{I} 20 q_{1}{ }^{3}+668 \mathrm{O} q_{1}{ }^{4}\right. \\
& \left.-34272 q_{1}{ }^{5}+{ }_{5} 6268 q_{1}{ }^{6}-\cdots \cdot\right] \\
& C_{2}=\left[\mathrm{I}+24 q_{1}+36 q_{1}{ }^{2}+384 q_{1}{ }^{3}+402 q_{1}{ }^{4}+3456 q_{1}{ }^{5}+3064 q_{1}{ }^{6}\right. \\
& \left.+23040 q_{1}{ }^{7}+18351 q_{1}^{8}+\cdots \cdot\right] \\
& -\mathrm{I} 2 q_{1} \log _{\mathrm{e}} \frac{\mathrm{I}}{q_{1}}\left[\mathrm{I}+2 q_{1}+24 q_{1}{ }^{2}+28 q_{1}{ }^{3}+264 q_{1}{ }^{4}+252 q_{1}{ }^{5}\right. \\
& \left.+2016 q_{1}^{6}+1696 q_{1}{ }^{7}+12264 q_{1}^{8}+\cdots \cdot\right]
\end{aligned}
$$


and the equation corresponding to (39) is ( $\alpha$ constant)

$$
\frac{\Delta q_{1}}{q_{1}}=\frac{\Delta z}{z} \cdot \frac{k^{\frac{s}{2}}}{4 \frac{a}{A}(\mathrm{I}-k)} \cdot \frac{z^{2}}{A^{2}}=\frac{\Delta z}{z} \frac{k^{\frac{5}{3}}(\mathrm{I}+k)}{4 \frac{a}{A} k^{\prime 2}} \cdot \frac{z^{2}}{A^{2}}=\frac{\Delta y k^{\frac{5}{3}}(\mathrm{I}+k) y^{2}}{4 \alpha k^{\prime 2}}
$$

Table 3 (appendix) gives the values of $\frac{\Delta z}{z}$ and $\frac{\Delta q}{q}$ or $\frac{\Delta q_{1}}{q_{1}}$ for a number of values of $\alpha$, and will be found useful in making calculations by this method.

In making a calculation of the value of $z_{\mathrm{m}}$, using these differential formulas, the procedure is as follows: Having obtained an approximate value of $z_{\mathrm{m}}$ by methods to be described below, the calculation of $q$ or $q_{1}$ is made (see Example 1 ), and the values thus found used in (33) or (37). In general, a somewhat more accurate value of $z_{\mathrm{m}}$ will thus be obtained, which will differ from the original by a certain fractional part of the whole, and this difference is then to be taken as $\frac{\Delta z}{z}$ in formula (39) or (42), according as (33) or (37) is being used. We obtain thus the fractional change in $q$ or $q_{1}$ corresponding to the second approximation of $z_{m}$. With this value of $\frac{\Delta q}{q}$ or $\frac{\Delta q_{1}}{q_{1}}$ we can at once compute from (38) or (40) a third approximation of $z_{m}$, and by repeating this process we rapidly approximate the true value of $z_{m}$. For this process we need calculate the coefficients in the differential formulas once only, since they do not need to be known with any great accuracy, and in general the values interpolated from Table 3 will be sufficient.

From an examination of this process it will be evident that each successive approximation of $z_{m}$ differs from the preceding by a fractional amount which is related to the corresponding fractional difference immediately preceding by a constant factor $f$, which is less than unity. Or, otherwise expressed, if $z_{1}, z_{2}$, ..... $z_{\mathrm{m}}$ are the successive approximations and

$$
\frac{\Delta z}{z}=\frac{z_{2}-z_{1}}{z_{1}} \text { then } \frac{z_{\mathrm{m}}-z_{1}}{z_{1}}=\frac{\Delta z}{z}\left[\mathrm{I}+f+f^{2}+\cdots+f_{\mathrm{m}}\right]=\frac{\Delta z}{z} \frac{\mathrm{I}}{\mathrm{I}-f} \text { in its }
$$

limit. The factor $f$ may be taken from Table 3 . 
Having obtained the final value of $z_{m}$, a check calculation should be made to see whether this value satisfies the main formula (33) or (37), which by the procedure just outlined does not therefore need to be used but twice, including the check calculation.

\section{APPROXIMATE FORMULAS}

Obviously the amount of time and labor necessary to obtain a precise value of $z_{m}$ by the preceding formulas depends, in large measure, on the closeness with which the first approximation, which is made the basis of the calculation, approaches the true value. It is, therefore, very desirable that, before using formulas (33) or (37), a value of $z_{m}$ may be found which is within, say, one part in a thousand of the truth.

The Rayleigh formula (8) or formula (9) is very satisfactory for this purpose for small values of $\frac{a}{A}$; for the important case of $\frac{a}{A}=\frac{\mathrm{I}}{2}$, it is, however, one per cent in error, and for still larger values of $\frac{a}{A}$ its insufficiency becomes such as to render its use entirely unsatisfactory.

For values of $\frac{a}{A}$ for which $a$ is nearly equal to $A$, the following development of (37) is of assistance.

Putting $\frac{a}{A}=\mathrm{I}-\delta, \frac{z}{A}=y$, we find from (6a) the approximation (holding for snall values of $\delta$ )

$$
q_{1}=\frac{\left(\delta^{2}+y^{2}\right)}{64}\left[\mathrm{I}+\delta+\frac{1}{8}\left(\delta^{2}+y^{2}\right)\right]
$$

and (37) may be written with sufficient accuracy

$$
y_{\mathrm{m}}{ }^{2}=\left(\frac{z_{m}}{A}\right)^{2}=32 \frac{a}{A} q_{1} \frac{\left[\mathrm{I}+24 q_{1}-\mathrm{I} 2 q_{1} \log _{\mathrm{e}} \frac{\mathrm{I}}{q_{1}}\right]}{\mathrm{I}-\mathrm{I} 6 q_{1}}
$$

To obtain a starting point for the use of (43) and (44), we neglect for the moment the last term in (43). The main term in (44) then becomes $y_{\mathrm{m}}{ }^{2}=32(\mathrm{I}-\delta) \frac{\left(\delta^{2}+y_{\mathrm{m}}{ }^{2}\right)}{64}(\mathrm{I}+\delta)$, whence

$$
y_{\mathrm{m}}=\delta \sqrt{\frac{\mathrm{I}-\delta^{2}}{\mathrm{I}+\delta^{2}}}
$$


Starting with this value in (43), we calculate a value of $q_{1}$ to be used in (44), and thus a second approximation of $y_{m}$, and so on. Very little work is necessary to obtain a very satisfactory approximation to $y_{\mathrm{m}}$, since the correction term in (44) need be calculated but once, and a very moderate accuracy in the value of $\log _{e} \frac{\mathrm{I}}{q_{1}}$ will suffice.

The range of usefulness of (43) and (44) is not, however, great, and does not include those cases which are most important in practice. For the especially interesting case, where $\frac{a}{A}$ is neither

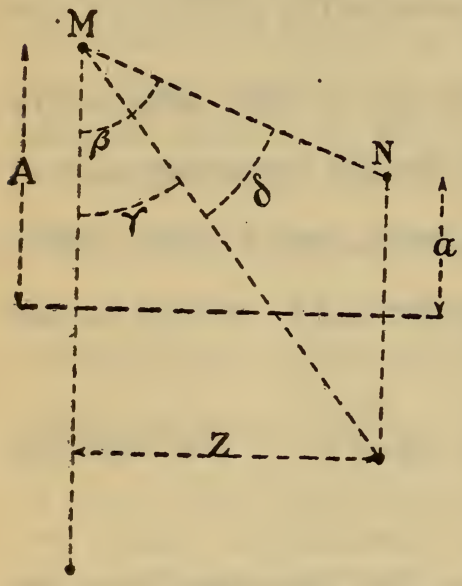

FIG. I.-Definition of the angle $\delta$

small nor near to unity, the following empirical method has proved very useful. Having, so far as the author has been able to determine, no simple physical significance, it is to be regarded as merely a useful coincidence.

If in Fig. I we pass a plane through the common axis of the two circles, and calculate the angle $\delta$, subtended by that diameter $N P$ of the small circle which lies in this plane (as viewed from the point $M$ of the larger circle, lying in the same plane), it will be found that the critical dis tance $z_{\mathrm{m}}$ has such a value as to make the angle $\psi=\frac{A}{a} \delta$ roughly the same, whatever the value of $\frac{a}{A}$.

This angle $\psi$ has a value of $45^{\circ}$ for $\alpha=\frac{a}{A}=\mathrm{r}$, increases to a value of about $46^{\circ} 30^{\prime}$ for $\alpha=0.7$, and falls with decreasing values of $\alpha$ to about $45^{\circ} 5 \mathrm{o}^{\prime}$ for $\alpha=0$.

-To aid in finding this angle for any given value of $\frac{a}{A}$, Table 4 will be useful. In this the angle $\psi$ has been calculated for a number of values of $\frac{a}{A}=\alpha$, for which the distance $z_{\mathrm{m}}$ was calculated to within a part in a million by the methods already 
described. To aid in interpolation the curve, Fig. 2, connecting the values in this table, has been plotted.

Having taken from the curve, or by interpolation from the table the value of the angle $\psi$ for any other value of $\alpha$ not included in the table, we derive the approximate value of $y_{\mathrm{m}}=\frac{z_{\mathrm{m}}}{A}$ desired, by means of the following equations.

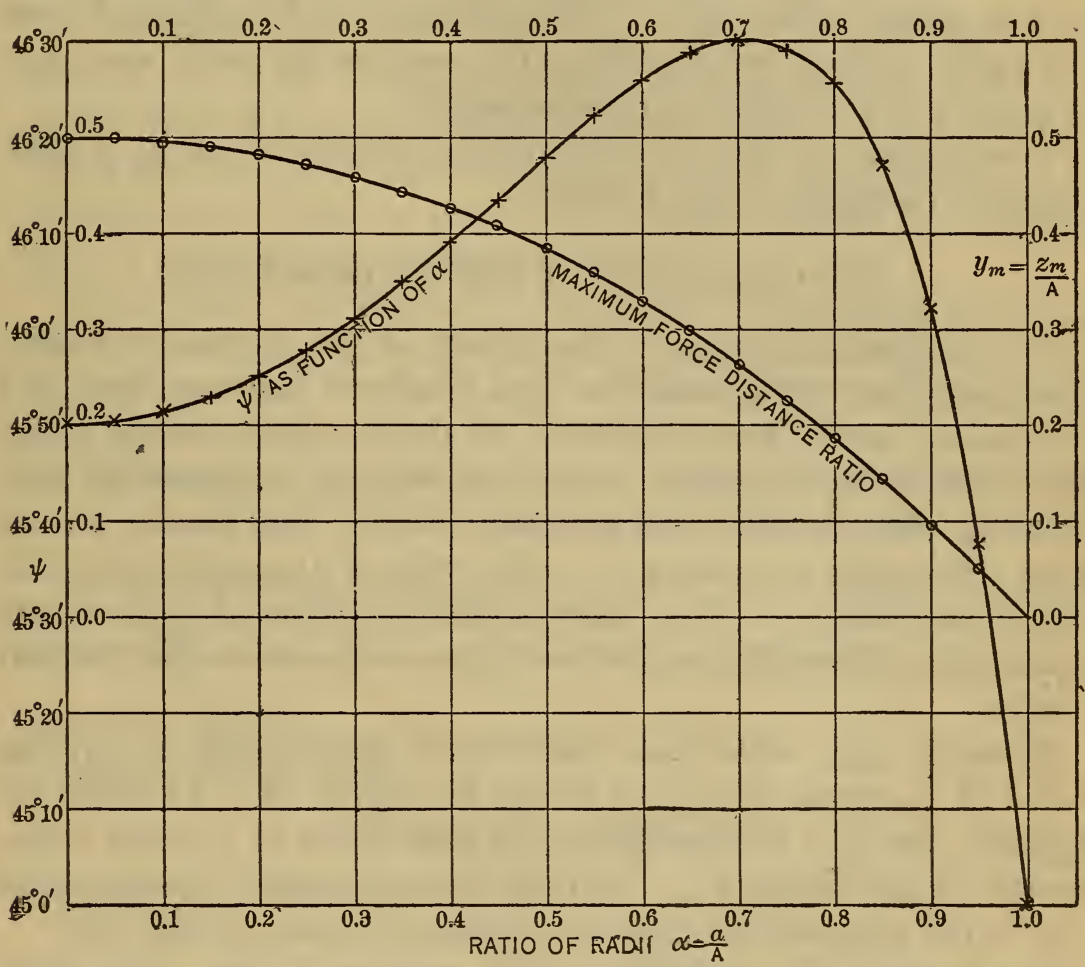

FIG. 2.-Giving the angle $\psi$ as a function of the ratio of the radii. Useful in obtaining

Putting a first approximation to $y_{m}$

$$
\begin{aligned}
\alpha & =\frac{a}{A}, \psi=\frac{\delta}{\alpha}, M=\tan \delta, \frac{z}{A}=y \\
M & =\tan (\beta-\gamma)=\frac{2 \alpha y}{\left(\mathrm{I}-\alpha^{2}\right)+y^{2}} \\
\therefore y_{\mathrm{m}} & =\frac{\alpha}{M}-\sqrt{\frac{\alpha^{2}}{M^{2}}-\left(\mathrm{I}-\alpha^{2}\right)}=\frac{\alpha}{M}\left[\mathrm{I}-\sqrt{\mathrm{I}-\frac{M^{2}}{\alpha^{2}}\left(\mathrm{I}-\alpha^{2}\right)}\right]
\end{aligned}
$$

the angles $\beta$ and $\gamma$ being those shown in Fig. I. 
A study of the equation

$$
\frac{\Delta y}{y}=\frac{\Delta M}{M}\left[\frac{\mathrm{I}}{\sqrt{\mathrm{I}-\frac{M^{2}}{\alpha^{2}}\left(\mathrm{I}-\alpha^{2}\right)}}\right] \quad\left(y=y_{\mathrm{m}}\right)
$$

shows, that in the most unfavorable case, to obtain $y_{\mathrm{m}}$ correct to one part in $\mathrm{r}, 000$, the angle $\psi$ taken from the curve must be correct to within about $30^{\prime \prime}$. This accuracy can be obtained from the curve without serious difficulty, even in the worst case, and in general this accuracy can be exceeded.

This method of obtaining a first approximation to $y_{\mathrm{m}}$ is illustrated in examples 4 and 5 below.

\section{THE CALCULATION OF THE MAXIMUM FORCE}

The distance $z_{\mathrm{m}}$ between the planes of the circular currents, corresponding to the condition that the force between them is a maximum, having been calculated by the formulas already given, the value of the maximum force itself may be computed by substituting the value of $z_{\mathrm{m}}$ in formula 5 or 6 . The former will be used principally for values of $\alpha$ (the ratio of the radii) lying between zero and 0.5 . The latter holds for circles of more nearly equal radii, although the two formulas overlap somewhat in their ranges.

Formula (34), which was derived by substituting in (5) the value of $y_{\mathrm{m}}$ given by (33), is useful for values of $\alpha$ to which (5) applies. In (34) the quantity $q_{\mathrm{o}}$ is that value of $q$ which corresponds to the distance $z_{\mathrm{m}}$. It must be emphasized, however, that (34) is not a general formula for the force, as are (5) and (6).

The method of calculation of the maximum force from these three formulas is illustrated in examples io and I , such a value of $\alpha$ having been chosen that all three formulas are applicable to the same case, thus giving a check on the accuracy of the three formulas.

Table 4 gives the values of $y_{\mathrm{m}}$ and the maximum force $F_{\mathrm{m}}$ for values of $\alpha$ from 0.05 to 0.95 in steps of 0.05 . In computing these values, seven place logarithms were used so that the values given should not be in error by more than a part in a million. 
Since, in a practical case, the coils would be designed so that their radii should approximate some round value, the data in Table 4 may be regarded as fundamental, and the usefulness of this table is further enhanced by the fact that the values of $y_{m}$ and $F_{\mathrm{m}}$ for values of $\alpha$ differing slightly from these fundamental values, may be obtained very simply from the data in the table without recourse to the main formulas (33) and (37).

For example, if a pair of coils, constructed to have radii in the ratio of $\mathrm{I}$ to 2 , are found, as a result of measurement, to have an actual ratio of the radii greater than one-half by 0.005 , the maximum force for this pair of coils can be found by applying to the value given in Table 4 for $\alpha=0.5$, a factor, which is a function both of the difference 0.005 and of $\alpha$.

To calculate this factor it is necessary to determine what relation exists between a small change in $\alpha$ and the changes in $y_{\mathrm{m}}$ and $F_{\mathrm{m}}$ corresponding. This matter is taken up in the next section.

\section{VARIATION FORMULAS FOR THE MAXIMUM FORCE $F_{m}$ AND THE MAXIMUM DISTANCE RATIO $y_{m}$}

To determine the change in $y_{\mathrm{m}}$ or $F_{\mathrm{m}}$ for a small fractional change $\frac{\Delta \alpha}{\alpha}$ in the ratio of the radii, we differentiate equations (33a) and (37a).

Equation (33a) gives

Writing

$$
\frac{\Delta y_{\mathrm{m}}}{y_{\mathrm{m}}}=\frac{\mathrm{I}}{2} \frac{\Delta \alpha}{\alpha}-\frac{\mathrm{I}}{2} \frac{\Delta q}{q}+\frac{\Delta P}{P}
$$

$$
\begin{gathered}
\Delta q=\frac{\partial q}{\partial \alpha} \Delta \alpha+\frac{\partial q}{\partial y_{\mathrm{m}}} \Delta y_{\mathrm{m}} \\
\frac{\Delta P}{P}=\frac{\mathrm{x}}{P} \frac{\partial P}{\partial q} \Delta q, \frac{\partial q}{\partial \alpha}=\frac{\partial q}{\partial k^{\prime}} \cdot \frac{\partial k^{\prime}}{\partial \alpha}, \frac{\partial q}{\partial y_{\mathrm{m}}}=\frac{\partial q}{\partial k^{\prime}} \cdot \frac{\partial k^{\prime}}{\partial y_{\mathrm{m}}}
\end{gathered}
$$

and using $(6 a)$ to evaluate the differential coefficients, we have

$$
\begin{gathered}
\Delta q=\frac{4 K^{\prime} \alpha}{Z}\left[y_{\mathrm{m}}{ }^{2} \cdot \frac{\Delta y_{\mathrm{m}}}{y_{\mathrm{m}}}-\frac{\lambda}{2} \cdot \frac{\Delta \alpha}{\alpha}\right] \\
\frac{\mathrm{I}}{P} \frac{\partial P}{\partial q}=-36 q\left[\mathrm{I}-\frac{37}{9} q^{2}+64 q^{4}-\frac{7 \mathrm{I} 65}{9} q^{6}+\frac{93974}{9} q^{8}-\cdots\right]
\end{gathered}
$$


which, substitued in (46) give the relation

$\frac{\Delta y_{\mathrm{m}}}{y_{\mathrm{m}}}\left[\mathrm{I}+\frac{4 K^{\prime} \alpha}{Z} y_{\mathrm{m}}{ }^{2}\left(\frac{\mathrm{I}}{2 q}-\frac{\mathrm{I}}{P} \frac{d P}{d q}\right)\right]=\frac{\mathrm{I}}{2} \frac{\Delta \alpha}{\alpha}\left[\mathrm{I}+\frac{4 K^{\prime} \alpha}{Z} \lambda\left(\frac{\mathrm{I}}{2 q}-\frac{\mathrm{I}}{P} \frac{d P}{d q}\right)\right]$

where

$$
\begin{aligned}
& K^{\prime}=-\frac{\left(\mathrm{I}+\mathrm{IO} q^{4}\right)}{2 \sqrt{k^{\prime}}\left(\mathrm{I}+\sqrt{k^{\prime}}\right)^{2}}, \lambda=\mathrm{I}-\alpha^{2}+y_{\mathrm{m}}^{2} \\
& Z=\left[(\mathrm{I}+\alpha)^{2}+y_{\mathrm{m}}^{2}\right]^{\frac{1}{2}} \sqrt{(\mathrm{I}-\alpha)^{2}+y_{\mathrm{m}}^{2}}
\end{aligned}
$$

For the change in the maximum force

$$
\frac{\Delta F_{\mathrm{m}}}{F_{\mathrm{m}}}=\frac{\mathrm{I}}{F_{\mathrm{m}}} \cdot \frac{\partial F_{\mathrm{m}}}{\partial q} \cdot \Delta q
$$

Writing $\frac{\Delta y_{\mathrm{m}}}{y_{\mathrm{m}}}=\frac{H}{2} \frac{\Delta \alpha}{\alpha}$ in the equation for $\Delta q$ previously derived, and differentiating (34) to obtain $\frac{\mathrm{I}}{F_{\mathrm{m}}} \frac{\partial F_{\mathrm{m}}}{\partial q}$, the final expression becomes

$\frac{\Delta F_{\mathrm{m}}}{F_{\mathrm{m}}}=\frac{4 K^{\prime} \alpha}{Z q}\left(y_{\mathrm{m}}^{2} H-\lambda\right)\left(\mathrm{I}+2 q^{2}+\mathrm{I} 24 q^{4}-\mathrm{II} 32 q^{6}+\mathrm{I} 4360 q^{8}-\cdots\right) \cdot \frac{\Delta \alpha}{\alpha}$

in which the factor $H$ is to be calculated by formula (48).

The expressions (48) and (5I) are to be used for values of $\alpha$ up to about 0.5. To cover the range of larger values, we start from (37a), which gives on differentiation

$$
\frac{\Delta y_{\mathrm{m}}}{y_{\mathrm{m}}}=\frac{\mathrm{I}}{2}\left[\frac{\Delta C_{2}}{C_{2}}-\frac{\Delta C_{1}}{C_{1}}+\frac{\Delta \alpha}{\alpha}+\frac{\Delta q_{1}}{q_{1}}\right]
$$

Writing

$$
\begin{array}{r}
\Delta q_{1}=\frac{\partial q_{1}}{\partial k} \cdot \frac{\partial k}{\partial \alpha} \cdot \Delta \alpha+\frac{\partial q_{1}}{\partial k} \cdot \frac{\partial k}{\partial y_{\mathrm{m}}} \cdot \Delta y_{\mathrm{m}} \\
K=-\frac{\mathrm{I}}{2 \sqrt{k}(\mathrm{I}+\sqrt{k})^{2}}, N=\frac{\Delta C_{1}}{C_{1}}=\frac{\mathrm{I}}{C_{1}} \cdot \frac{\partial C_{1}}{\partial q_{1}} \cdot \Delta q_{1} \\
Y=\left[(\mathrm{I}+\alpha)^{2}+y_{\mathrm{m}}^{2}\right]^{\frac{3}{2}}, M=\frac{\Delta C_{2}}{C_{2}}=\frac{\mathrm{I}}{C_{2}} \cdot \frac{\partial C_{2}}{\partial q_{1}} \cdot \Delta q_{1}
\end{array}
$$


and using (6a) to carry out the differentiations indicated in (53),

$$
\Delta q_{1}=\frac{K \sqrt{\alpha}}{Y}\left[\lambda \frac{\Delta \alpha}{\alpha}-2 y_{\mathrm{m}}^{2} \cdot \frac{\Delta y_{\mathrm{m}}}{y_{\mathrm{m}}}\right]
$$

from whence we find immediately

$$
\frac{\Delta y_{\mathrm{m}}}{y_{\mathrm{m}}}\left[\mathrm{I}+\frac{K \sqrt{\alpha}}{Y} y_{\mathrm{m}}{ }^{2}\left(M-N+\frac{\mathrm{I}}{q_{1}}\right)\right]=\frac{\mathrm{I}}{2} \frac{\Delta \alpha}{\alpha}\left[\mathrm{I}+\frac{K \sqrt{\alpha}}{Y} \lambda\left(M-N+\frac{\mathrm{I}}{q_{1}}\right)\right]
$$

in which the quantities $M$ and $N$ are to be calculated from (53) and (4I).

Equation (6) for the maximum force may be written $F_{m}=\frac{\pi y_{m} \Phi}{16 q_{1} \sqrt{\alpha}}$ which gives on differentiation

$$
\frac{\Delta F_{\mathrm{m}}}{F_{\mathrm{m}}}=\frac{\Delta y_{\mathrm{m}}}{y_{\mathrm{m}}}-\frac{\mathrm{I}}{2} \frac{\Delta \alpha}{\alpha}-\frac{\Delta q_{1}}{q_{1}}+\frac{\Delta \Phi}{\Phi}
$$

Putting

$$
\frac{\Delta \Phi}{\Phi}=\frac{\mathrm{I}}{\Phi} \frac{d \Phi}{d q_{1}} \cdot \Delta q_{1}=\Pi \Delta q_{1}
$$

and writing as before, $\frac{\Delta y_{\mathrm{m}}}{y_{\mathrm{m}}}=\frac{H}{2} \cdot \frac{\Delta \alpha}{\alpha}$, equations (56) and (57) become

$$
\begin{aligned}
\frac{\Delta F_{\mathrm{m}}}{F_{\mathrm{m}}} & =\frac{\mathrm{I}}{2}(H-\mathrm{I}) \frac{\Delta \alpha}{\alpha}+\left(\Pi-\frac{\mathrm{I}}{q_{1}}\right) \Delta q_{1} \\
& =\frac{\Delta \alpha}{\alpha}\left[\frac{\mathrm{I}}{2}(H-\mathrm{I})+\left(\Pi-\frac{\mathrm{I}}{q_{1}}\right) \frac{K \sqrt{\alpha}}{Y}\left(\lambda-H y_{\mathrm{m}}{ }^{2}\right)\right]
\end{aligned}
$$

To calculate $\Pi$ we have

$$
\begin{aligned}
\frac{d \Phi}{d q_{1}}= & 24\left(\mathrm{I}-2 \mathrm{I} q_{1}+\mathrm{I} 84 q_{1}{ }^{2}-\mathrm{I} \mathrm{I} 089 q_{1}{ }^{3}+5 \mathrm{I} 60 q_{1}{ }^{4}-\cdots\right) \\
& -\mathrm{I} 2 \log _{e} \frac{\mathrm{I}}{q_{1}} \cdot\left(\mathrm{I}-20 q_{1}+\mathrm{I} 80 q_{1}{ }^{2}-\mathrm{I} 200 q_{1}{ }^{3}+6500 q_{1}{ }^{4}-\cdots\right) \\
\Phi= & \left(\mathrm{I}+\mathrm{I} 2 q_{1}-\mathrm{I} 92 q_{1}{ }^{2}+\mathrm{I} 232 q_{1}{ }^{3}-5634 q_{1}{ }^{4}+\cdots\right) \\
& -\mathrm{I} 2 q_{1} \log _{\mathrm{e}} \frac{\mathrm{I}}{q_{1}} \cdot\left(\mathrm{I}-\mathrm{IO} q_{1}+60 q_{1}{ }^{2}-300 q_{1}{ }^{3}+\mathrm{I} 300 q_{1}{ }^{4}-\cdots\right)
\end{aligned}
$$


These formulas, although somewhat complicated in appearance, are not difficult to use, especially since most of the quantities involved enter also in the main formulas.

The factors $\eta$ and $\epsilon$, defined by the equations

$$
\begin{aligned}
\frac{\Delta y_{\mathrm{m}}}{y_{\mathrm{m}}} & =\eta \frac{\Delta \alpha}{\alpha} \\
\frac{\Delta F_{\mathrm{m}}}{F_{\mathrm{m}}} & =\epsilon \frac{\Delta \alpha}{\alpha}
\end{aligned}
$$

have been calculated by means of the equations just developed, for the same values of $\alpha$ as in Table 4 , and are given in Table 5 . Values of $\eta$ and $\epsilon$ for other values of $\alpha$ may be derived by interpolation. From the method of derivation of the formulas for $\eta$ and $\epsilon$, it is evident that these values hold only for very small values of $\frac{\Delta \alpha}{\alpha}$. For differences, $\frac{\Delta \alpha}{\alpha}$, greater than about $0.00 \mathrm{I}$, the values taken from the table must be corrected by a factor whose value is determined from the following considerations.

Table 4 gives the value $F_{0}$ of the maximum force for a certain value $\alpha_{0}$ of the ratio of the radii. It is desired to find the value of the maximum force $F_{1}=F_{0}\left(\mathrm{I}+\frac{\Delta F}{F_{0}}\right)$ corresponding to a slightly different ratio of the radii $\alpha_{1}$, connected with the former ratio by the relation $\alpha_{1}=\alpha_{0}\left(\mathrm{I}+\frac{\Delta \alpha}{\alpha_{0}}\right)$.

For an infinitely small increment of $\alpha_{0}, d \alpha$, the fractional change in the value of the maximum force is $\frac{d F}{F_{0}}=\epsilon_{0} \frac{d \alpha}{\alpha_{0}}$, the value of $\epsilon_{0}$ being that taken from Table 5 for the argument $\alpha_{0}$. Similarly, in the neighborhood of $\alpha_{1}, \frac{d F}{F_{1}}=\epsilon_{1} \frac{d \alpha}{\alpha_{1}}, \epsilon_{1}$ being obtained from Table 5 by direct interpolation, using the argument $\alpha_{1}$. For the finite difference $\Delta \alpha=\alpha_{1}-\alpha_{0}$, the corresponding difference $\Delta F$ is

$$
\frac{\Delta F}{F_{0}}=\frac{I}{F_{0}} \int_{\alpha=\alpha_{0}}^{\alpha=\alpha_{1}} d F=\frac{I}{\alpha_{0}} \int_{\alpha_{0}}^{\alpha_{1}} \epsilon d \alpha
$$

where the coefficient $\epsilon$ is a function of $\alpha$. We may approximate 
the value of this integral closely, if we use the average value of $\epsilon$ over the interval $\Delta \alpha$. This average value is, however, not the simple mean of $\epsilon_{0}$ and $\epsilon_{1}$, since $\epsilon_{1}=\frac{\alpha_{1}}{F_{1}} \frac{d F}{d \alpha}$ and that portion of the integral contributed by the element $d \alpha$ in the neighborhood of $\alpha_{1}$ is not $\epsilon_{1} \frac{d \alpha}{\alpha_{1}}$, but $\epsilon_{1} \frac{\alpha_{0}}{\alpha_{1}} \frac{F_{1}}{F_{0}}$ because in the original statement of the problem, fractional increments of $F_{\mathrm{m}}$ and $\alpha$ are to be referred to $F_{0}$ and $\alpha_{0}$, the fundamental values given in Table 4 .

Taking these facts into account in finding the average value of $\epsilon$, we find as an approximation to the integral the expression

$$
\frac{\Delta F}{F_{0}}=\frac{1}{2} \frac{\Delta \alpha}{\alpha_{0}}\left[\epsilon_{0}+\frac{F_{1}}{F_{0}} \cdot \frac{\alpha_{0}}{\alpha_{1}} \cdot \epsilon_{1}\right]
$$

which is a very close approximation to the truth for values of $\frac{\Delta \alpha}{\alpha_{0}}$ as great as o.or, over the range of values of $\alpha$ for which interpolation in Table 5 may be accurately made.

Entirely similar considerations enable to be found for the change in $y_{\mathrm{m}}$ corresponding to a small change in $\alpha$, the equation

$$
\frac{\Delta y}{y_{0}}=\frac{\mathrm{I}}{2} \frac{\Delta \alpha}{\alpha_{0}}\left[\eta_{0}+\frac{y_{1}}{y_{0}} \cdot \frac{\alpha_{0}}{\alpha_{1}} \cdot \eta_{1}\right]
$$

These formulas are illustrated in examples $\mathrm{I}_{3}$ and $\mathrm{I} 4$, where the constants are calculated for the coils of the Bureau of Standards current balance, and the results compared with the values calculated by the methods described in the original article.

\section{VARIATION OF THE FORCE FOR SMALL DISPLACE- MENTS OF THE COILS FROM THE POSITION OF MAXIMUM FORCE}

From Maxwell's formula (3) it is evident that the force corresponding to positions of the coils in the neighborhood of the critical distance bears to the maximum value of the force a relation which may be expressed by the power series

$$
F=F_{\mathrm{m}}\left[\mathrm{I}-\gamma\left(z_{\mathrm{m}}-z\right)^{2}+\delta\left(z_{\mathrm{m}}-z\right)^{3}-\cdots\right]
$$

in which $z$ is the actual distance between the planes of the coils, $z_{\mathrm{m}}$ the distance for which the force has its maximum value $F_{\mathrm{m}}$, 
and $\gamma$ and $\delta$ are constants. The term in the first power of the displacement $\left(z_{\mathrm{m}}-z\right)$ is lacking.

Remembering that the force is a function of the ratio of the radii alone, we may write

$$
\frac{\Delta F}{F_{\mathrm{m}}}=-c\left(\frac{\Delta y}{y_{\mathrm{m}}}\right)^{2}+d\left(\frac{\Delta y}{y_{\mathrm{m}}}\right)^{3}-\cdots \cdot
$$

where $c$ and $d$ are positive constants depending on $\alpha$ alone. This formula holds with a good deal of accuracy for values of $\frac{\Delta y}{y_{m}}$ as great as 0.04 .

The following table will allow of the interpolation of the value of the constant $c$ with an accuracy sufficient in practically all cases:

Values of the Constant $c$ as a Function of $\alpha$

\begin{tabular}{|c|c|}
\hline$\alpha$ & $c$ \\
\hline 0.1 & 0.805 \\
0.3 & 0.755 \\
0.5 & 0.690 \\
0.7 & 0.610 \\
0.9 & 0.510 \\
\hline
\end{tabular}

The constant $d$ is sensibly equal to 0.5 for values of $\alpha$ ranging from o.I to 0.9. These values of $c$ and $d$ were found by actually calculating the force, by the methods already developed, for various values of $\alpha$, and for differences $\frac{\Delta y}{y_{m}}$ of \pm 0.01 and \pm 0.04 . The value of $\frac{\Delta F}{F_{\mathrm{m}}}$ corresponding to a negative displacement $\frac{-\Delta y}{y_{\mathrm{m}}}$ differs from that produced by an equal positive displacement $\frac{\Delta y}{y_{\mathrm{m}}}$ by an amount which depends on $d$ alone. The average value of $\frac{\Delta F}{F_{\mathrm{m}}}$ corresponding to equal positive and negative displacements $\frac{\Delta y}{y_{m}}$ and $\frac{-\Delta y}{y_{m}}$ is a measure of the value of $c$ alone. The values of $c$ and $d$ here given show how slowly. the force varies in the neighborhood of the position of maximum force. 


\section{BEST VALUES OF RADII OF THE COILS}

The formulas and methods above developed furnish a solution of the general problem of finding the maximum force between two circular currents with any given ratio of their radii. The practically important question, What ratio of the radii is best in designing a Rayleigh current balance? may also be answered in a general way from a consideration of the numerical values in the tables here presented.

The conditions which should be fulfilled by a current balance are broadly as follows:

I. The force should be large enough to be accurately measurable.

2. The number of turns on the coils should be kept as small as possible in order to provide that the resistance of the coils may be small. Otherwise the heating of the coils may cause considerable changes in the dimensions of the coils, as well as air currents which will affect the accuracy of the weighing.

3. The cross section of the coils should be made small relatively to the distance between the windings of the fixed and movable coils.

Naturally the best design will be one of compromise, since these conditions are to some extent incompatible; and, as is usual, quite a latitude in the design is allowable without materially detracting from the performance of the balance.

Large values of $\alpha$ (radius of the moving coil nearly equal to that of the fixed coil) give large values of the force per turn per unit current. This advantage; however, is counterbalanced by the necessity of keeping the cross section of the coils small; for, as may be seen from Table 4 , the distance $z_{\mathrm{m}}$ is, in the cases supposed, so small that very moderate values of the area of cross section will give rise to large corrections for the latter, with consequent difficulty in determining its value. Further, the change in the force for small changes in the radii and for small displacements of the coils from the position of maximum force are much larger with coils of nearly equal radii ( $\alpha$ large) than for coils of quite different radii ( $\alpha$ small).

$18423^{\circ}-16-3$ 
These disadvantages, combined with the undesirability of using such large moving coils as postulate a long arm balance, render impracticable the employment of moving coils with radii nearly equal to the radius of the fixed coil.

The opposite case of a balance whose moving coil is small relatively to the fixed coil, although free from the difficulties of the previous case, suffers under the disadvantage of a small value of the force per unit current per turn, so that either the number of turns on the coils or else the value of the currents must be increased to compensate for this difficulty. This renders probable in the former case a troublesome correction for the area of cross section of the coils, and in the latter difficulties in the manipulation of the balance, as a result of air currents or, at least, considerable changes in the dimensions of the coils as a result of undue heating of the coils.

From these considerations one is lead to expect that a suitable design will avoid either extreme, and this is confirmed by the numerical results given in Table 4 . Starting with very small values of the maximum force (per unit current per turn in each coil) for values of $\alpha$ about O.I, the value of $F_{\mathrm{m}}$ increases rapidly with increase of $\alpha$, so that it is not difficult to obtain a sufficiently large value of the force with a moving coil having a radius about one-half as large as that of the fixed coils, and without encountering serious difficulty from the disadvantages which beset the use of relatively small moving coils on the one hand and relatively large moving coils on the other.

These points are illustrated in the following example, in which are tabulated the principal constants of five current balances, each of which is supposed to have a fixed coil of $25 \mathrm{~cm}$ radius, the radii of the moving coils in the different cases being taken of various values. The nomenclature is the same as in the preceding discussion, except that there are introduced the additional quantities $d_{\mathrm{m}}$, the distance between the windings of the coils in a radial plane and $\Delta$ the fractional change in the value of the force for a displacement of $\mathrm{I} \mathrm{mm}$ from the position of maximum force. 


\begin{tabular}{|c|c|c|c|c|c|}
\hline$\alpha=$ & 0.1 & 0.3 & 0.5 & 0.7 & 0.9 \\
\hline a $\ldots \ldots \ldots \ldots \ldots \ldots \ldots \ldots \ldots$ & 2.5 & 7.5 & 12.5 & 17.5 & 22.5 \\
\hline$z_{m} \ldots \ldots \ldots \ldots \ldots \ldots \ldots \ldots \ldots \ldots \ldots \ldots \ldots \ldots$ & 12.39 & 11.47 & 9.59 & 6.61 & 2.446 \\
\hline 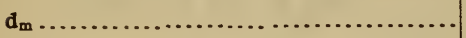 & 25.68 & 20.92 & 15.75 & 10.00 & 3.50 \\
\hline 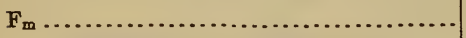 & 0.1709 & 1.646 & 5.352 & 14.507 & 58.06 \\
\hline$\varepsilon \ldots \ldots \ldots \ldots \ldots \ldots \ldots \ldots \ldots \ldots \ldots \ldots \ldots \ldots \ldots \ldots \ldots \ldots \ldots \ldots$ & 2.016 & 2.160 & 2.549 & 3.649 & 9.934 \\
\hline 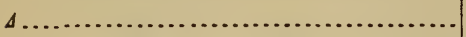 & $53 \times 10^{-6}$ & $60 \times 10^{-6}$ & $75 \times 10^{-6}$ & $137 \times 10^{-6}$ & $868 \times 10^{-6}$ \\
\hline
\end{tabular}

An examination of these values shows that it will not be advisable to use a value of $\alpha$ greater than about 0.7 nor less than about o.3. The coils of the Bureau of Standards coils were designed to have ratios of 0.4 and 0.5 .

\section{EXAMPLES}

\section{EXAMPLE 1. CALCULATION OF $q$ AND $q_{1}$ (FORMULAE 6a)}

One or both of these quantities has to be calculated when the majority of the formulas of this paper are to be used. The following arrangement of the calculations has been found convenient:

Let us obtain the values of $q$ and $q_{1}$ corresponding to the case $\alpha=\frac{a}{A}=0.5, y=\frac{z}{A}=0.3875$. The formulas (6a) for $k$ and $k^{\prime}$ may be conveniently written in the form

$$
\begin{aligned}
& k^{2}=\frac{4 \frac{a}{A}}{\left(\mathrm{I}+\frac{a}{A}\right)^{2}+\left(\frac{z}{A}\right)^{2}}=\frac{4 \frac{a}{A}}{4 \frac{a}{A}+\left[\left(\mathrm{I}-\frac{a}{A}\right)^{2}+\frac{z^{2}}{A^{2}}\right]}=\frac{4 \alpha}{4 \alpha+\left[(\mathrm{I}-\alpha)^{2}+y^{2}\right]} \\
& k^{\prime 2}=\frac{\left(\mathrm{I}-\frac{a}{A}\right)^{2}+\left(\frac{z}{A}\right)^{2}}{\left(\mathrm{I}+\frac{a}{A}\right)^{2}+\left(\frac{z}{A}\right)^{2}}=\frac{\left(\mathrm{I}-\frac{a}{A}\right)^{2}+\left(\frac{z}{A}\right)^{2}}{4 \frac{a}{A}+\left[\left(\mathrm{I}-\frac{a}{A}\right)^{2}+\frac{z^{2}}{A^{2}}\right]}=\frac{\left[(\mathrm{I}-\alpha)^{2}+y^{2}\right]}{4 \alpha+\left[(\mathrm{I}-\alpha)^{2}+y^{2}\right]}
\end{aligned}
$$

Here $4 \alpha=2$,

$$
\begin{aligned}
& (\mathrm{I}-\alpha)^{2}=0.25 \log y=\overline{\mathrm{I}} .58827 \mathrm{I} 7 \\
& 2 \log y=\overline{\mathrm{I}} \cdot \mathrm{I}_{7} 65434 \\
& y^{2}=0.15 \text { OI } 5624 \\
& (\mathrm{I}-\alpha)^{2}=0.25 \\
& \text { Sum }=\overline{0.40015624} \\
& k^{2}=\frac{2}{2.40015624} \\
& k^{\prime 2}=\frac{0.40015624}{2.40015624}
\end{aligned}
$$


$\log$ numerator $=0.3010300$

$\log$ denominator $=0.3802395$

$\log k^{2}=\overline{\overline{\mathrm{I}} .9207905}$

$\log M=0.878885 \mathrm{I}$

Diff. $=\log \frac{l}{2}=\overline{\mathrm{I} .0419054}$

$$
\begin{aligned}
5 \log \frac{l}{2} & =\overline{5} .20952 \\
\log 2 & =0.30103 \\
\text { Sum } & =\overline{\overline{5} .51055} \\
2\left(\frac{l}{2}\right)^{5} & =0.00003240 \\
\frac{l}{2} & =0.11012995 \\
q & =0.1 \text { I016235 } \\
\log q & =\overline{\mathrm{r}} .0420332
\end{aligned}
$$

Check.

$$
\begin{array}{rlrl}
\sqrt{k^{\prime}} & =0.6389950 & & \\
\mathrm{I}-\sqrt{k^{\prime}} & =0.3610050 & \log \left(\mathrm{I}-\sqrt{k^{\prime}}\right) & =\overline{\mathrm{I}} .5575 \mathrm{I} 32 \\
2\left(\mathrm{I}+\sqrt{k^{\prime}}\right) & =3.2779900 & \log 2\left(\mathrm{I}+\sqrt{k^{\prime}}\right) & =0.5 \mathrm{I} 56076 \\
& \text { Diff. }=\log \frac{l}{2}=\overline{\mathrm{I}} .04 \mathrm{I} 9056
\end{array}
$$

$\log$ numerator $=\overline{\mathrm{r}} .6022296$

$\log$ denominator $=0.3802395$ $\log k^{\prime 2}=\overline{\mathrm{I} .22 \mathrm{I} 990 \mathrm{I}}$

$\log k^{\prime}=\overline{\mathrm{I}}$.6109950

$\log , \sqrt{k^{\prime}}=\overline{\mathrm{I}} .8054975$

$$
\left(\mathrm{I}+\sqrt{k^{\prime}}\right)=\mathrm{I} .6389950
$$$$
\left(\mathrm{I}+k^{\prime}\right)=\mathrm{I} .4083 \mathrm{I} 47
$$$$
\log \left(\mathrm{I}+\sqrt{k^{\prime}}\right)=0.2145777
$$

$\log \left(\mathrm{I}+k^{\prime}\right)=0.1486997$

$\log 2=0.3010300$

$\log 2\left(\mathrm{I}+k^{\prime}\right)=0.4497297$

$2 \log \left(\mathrm{I}+\sqrt{k^{\prime}}\right)=\underline{0.4291554}$

$\log M=\operatorname{Sum}=0.878885 \mathrm{I}$

In the above calculation $q$ has been obtained by the use of the second of the formulas under $(6 \mathrm{a})$. The first relation, that involving. I $-\sqrt{k^{\prime}}$, may, when $\sqrt{k^{\prime}}$ is nearly equal to unity, give an accuracy much inferior to that obtained by the other formula. It is, however, useful as a check, and is so used in the calculation just given.

The procedure to be followed in calculating $q_{1}$ is, in every respect, similar to that for $q$, except that $k$ takes the place of $k^{\prime}$ throughout, and that the difference between $q_{1}$ and $\frac{l_{1}}{2}$ is, on account of the smallness of $q_{1}$, generally negligible. 


$$
\begin{aligned}
& \log k^{2}=\overline{\mathrm{r}} .9207905 \\
& \log k^{\prime 2}=\overline{\mathrm{I}} .2219901 \\
& \log k=\overline{\mathrm{r}} .9603952 \\
& \log M=\text { I.I65192 I } \\
& \log \sqrt{k}=\overline{\mathrm{r}} .9801976 \\
& \mathrm{I}+\sqrt{k}=\mathrm{I} .9554272 \\
& \mathrm{I}+k=\mathrm{I} .9 \mathrm{I} 284 \mathrm{II} \\
& \log (\mathrm{I}+\sqrt{k})=0.2912416 \\
& \log (\mathrm{I}+k)=0.28 \mathrm{I} 6789 \\
& \log 2=0.3010300 \\
& \log 2(\mathrm{I}+k)=\overline{0.5827089} \\
& 2 \log (\mathrm{I}+\sqrt{k})=\underline{0.5824832} \\
& \log \frac{l_{1}}{2}=\log q=\overline{\overline{2} .0567980} \\
& \text { since } 5 \log \frac{l_{1}}{2}=\overline{\mathrm{I}} \overline{0} .28399 \\
& q_{1}=0.011397 \text { I } 95 \\
& \log M=\text { Sum }=\overline{\text { I.I65I92I }} \\
& \sqrt{k}=0.9554272 \\
& \mathrm{I}-\sqrt{k}=0.0445728 \\
& 2(\mathrm{I}+\sqrt{k})=\underline{3.9108544} \\
& \log (\mathrm{I}-\sqrt{k})=\overline{\mathbf{2}} .6490699 \\
& \log 2(\mathrm{I}+\sqrt{k})=0.59227 \mathrm{I} 6 \\
& \text { Sum }=\log \frac{l_{1}}{2}=\overline{2} .0567983
\end{aligned}
$$

EXAMPLE 2.-ILLUSTRATING THE CALCULATION OF $y_{\mathrm{m}}=\frac{z_{\mathrm{m}}}{A}$ BY (33), STARTING FROM THE APPROXIMATION BY RAYLEIGH'S FORMULA (8)

For the case $\alpha=\frac{a}{A}=0.5$ we obtain as a first approximation $y=0.3875$. The value of $q$ corresponding to this value of $y$ has been calculated in the previous example.

From Table I we find by interpolation that the value of $P$ corresponding to $q=0.11016235$ is $P=0.807662$. The calculation of a second approximation to $y$ by equation (33) is as follows:

$$
\begin{array}{rlrl}
\log q & =\overline{\mathrm{I}} .0420332 & \frac{1}{2} \log \frac{\alpha}{20 q} & =\overline{\mathrm{I}} .6779534 \\
\log 2 \mathrm{O} & =\underline{\mathrm{I} .3010300} & \log P & =\overline{\mathrm{I}} .9072297 \\
\operatorname{Sum} & =\overline{0.3430632} & \text { Sum }=\log y & =\overline{\mathrm{I}} \cdot 585 \mathrm{I} 83 \mathrm{I} \\
\log \alpha & =\overline{\mathrm{I}} .6989700 & y & =0.384754= \\
\log \frac{\alpha}{20 q} & =\overline{\mathrm{I}} \cdot 3559068 & \begin{aligned}
\text { second approximation }
\end{aligned}
\end{array}
$$

The calculation may now be repeated, using this second approximation for $y_{\mathrm{m}}$ to find more accurate values of $k, k^{\prime}$, and $q$, and thence a third approximation to $y_{\mathrm{m}}$, and so on till successive 
approximations do not differ sensibly from the preceding. The successive approximations in the present case are, to six places of logarithms,

$\begin{array}{cc}\text { Approximation } & \log y \\ \text { I } & \overline{\mathrm{I}} .588272 \\ 2 & \overline{\mathrm{I}} .585 \mathrm{I} 83 \\ 3 & \overline{\mathrm{I}} .584228 \\ 4 & \overline{\mathrm{I}} .583933 \\ 5 & \overline{\mathrm{I}} .583843 \\ 6 & \overline{\mathrm{I}} .583816 \\ 7 & \overline{\mathrm{I}} .583807 \\ 8 & \overline{\mathrm{I}} .583804 \\ 9 & \overline{\mathrm{I}} .583803\end{array}$

The necessary labor may, however, be very much reduced by the use of the differential formulas (38) and (39). Using in (39) the second approximation of $y_{\mathrm{m}}$ already found, and the values of $k, k^{\prime}$, and $q$ already calculated above, there results

$$
\frac{\Delta q}{q}=-0.3330 \frac{\Delta z}{z} \quad, \quad \frac{\Delta z}{z}=-0.9215 \frac{\Delta q}{q}
$$

Since we are concerned with fairly small differences, we may, without serious error, use in place of $\frac{\Delta q}{q}$ and $\frac{\Delta z}{z}$ the differences in the logarithms of the different values of $q$ and $z$ or $y$. The difference of the logarithms of the first. and second approximation to $y_{\mathrm{m}}$ is 0.0030866 . The formulas (38) and (39) show that the logarithm of the third approximation to $y_{\mathrm{m}}$ will differ from that of the second approximation by 0.3330 of 0.9215 of 0.0030886 , and the difference between the logarithms of the fourth and third approximations will be less than this value in the ratio of the same factor, and so on for each successive approximation. Denoting this factor by $f$, we may find at once the factor $F$ by which we must multiply the difference between the first and second approximations, in order to obtain the amount by which the final value differs from the first approximation. It is $F=\mathrm{I}+f+f^{2}+f^{3}+\ldots+f^{n}$, which gives, when $n$ is taken indefinitely great, the value $F=\frac{I}{I-f}$. Using here the value $f=0.30685$, 
and the difference 0.0030866 , the final difference 0.0044559 is found, which subtracted from the logarithm of the first approximation gives $\log y_{\mathrm{m}}=\overline{\mathrm{I}} \cdot 583^{8} \mathrm{I}_{5} 8$. This value differs slightly from the true value, for the reason that the data used in calculating the factor $f$ does not quite apply to such a large interval as that here in question. We may, however, use this value as the starting point in obtaining the true value. Using the value $\overline{\mathrm{r}} .5838 \mathrm{I} 58$ as a first approximation, formula (33) gives as a second approximation $\overline{\mathrm{r}} .5838076$ (a difference in the logarithm of -82 in the seventh place), which, using the same value of the factor $f$ as before, indicates that the correction to be applied is - I 8 in the seventh place, and that the final value is $\overline{\mathrm{I}} .5838040$. Substituting this value in formula (33) the result obtained does not differ from this by as much as a unit in the seventh place, showing that equation (33) is satisfied and that the correct value has been found.

This method was used in obtaining the data in Table 4. In most cases, however, the first approximation used did not differ by more than one or two parts in a thousand from the true value, and for such cases a single application of formula (33) was necessary, excepting as a check on the results by the differential formula. To aid in further calculations, the data of Table 3 have been calculated, and by interpolation from this table it should be possible to derive the proper value of $f$ without recourse to formulas (38) and (39).

\section{EXAMPLE 3.-CALCULATION OF $y_{\mathrm{m}}$ BY FORMULA (37)}

To illustrate the use of formula (37) we may take the same case as that in the preceding example, but will start from the final value found in the latter, and show that it is consistent with (37). The mode of procedure is, however, the same, in case only a first approximation is available, except that the factor $f$ is to be calculated from the differential formulas (40) and (42).

Starting from the value $\log y=\overline{\mathrm{I}} \cdot 5838040$, we find for $q_{1}$ the value 0.01131756 , to which corresponds the value $X=0.8$ I2335, which is obtained by interpolation in Table 2. Direct calculation of $X$ from equation (37) gives the value 0.8123349 (see example 7). 
The calculation of $y_{\mathrm{m}}$ by formula (37) is then as follows:

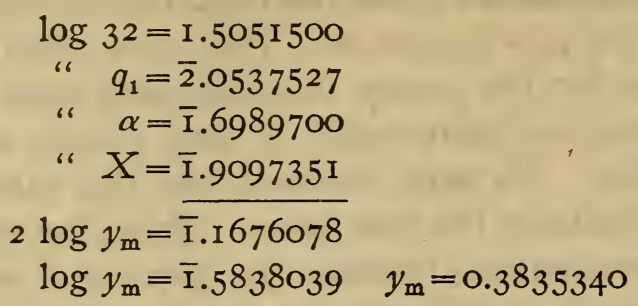

The value of the logarithm of $y_{m}$ just found agrees as closely with that found by (33) as the accuracy of the logarithms will allow. The results by the two formulas agree within, at most, one part in four million. This case must be regarded as giving a very searching test of the accuracy of the coefficients in the two formulas, since terms of the eighth order have to be taken into account in each of the formulas.

EXAMPLE 4.-ILLUSTRATION OF THE USE OF CURVE (FIG. 2) FOR OBTAINING A FIRST APPROXIMATION

Let us take the value $\alpha=0.45$. For this value of $\alpha$ the curve, Fig. 2, gives

$$
\begin{aligned}
& \psi=\frac{\delta}{a}=46^{\circ} 13^{\prime} .6 \text { or } 46^{\circ} 13^{\prime} 36^{\prime \prime} \\
& \delta=46^{\circ} 13^{\prime} 36^{\prime \prime} \times 0.45=20^{\circ} 48^{\prime} 7^{\prime \prime} .2 \\
& \log \alpha=\log 0.45=\overline{\mathrm{I}} .6532125 \\
& \text { " } M=\text { " } \tan \delta \quad=\overline{\mathrm{I}} .5796743 \\
& \mathrm{I}-\alpha^{2}=\mathrm{I}-0.2025 \\
& \text { " } \quad \underset{M}{\alpha}=0.0735382 \\
& 2 \text { " " }=0.1470764 \\
& \text { " }\left(\mathrm{I}-\alpha^{2}\right)=\overline{\mathrm{I}} .9017307 \\
& \therefore{ }^{\prime \prime} \frac{M^{2}}{a^{2}}\left(\mathrm{I}-\alpha^{2}\right)=\overline{\mathrm{I}} .7546543 \quad \frac{M^{2}}{\alpha^{2}}\left(\mathrm{I}-\alpha^{2}\right)=0.5684003 \\
& \log \left[\mathrm{I}-\frac{M^{2}}{\alpha^{2}}\left(\mathrm{I}-\alpha^{2}\right)\right]=\overline{\mathrm{I}} .63508 \mathrm{II} \\
& \mathrm{I}-\frac{M^{2}}{\alpha^{2}}\left(\mathrm{I}-\alpha^{2}\right)=0.4315997 \\
& \frac{1}{2} \text { “ } \\
& =\overline{\mathrm{I}} .8 \mathrm{I} 75406 \\
& \sqrt{\mathrm{I}-\frac{M^{2}}{\alpha^{2}}\left(\mathrm{I}-\alpha^{2}\right)}=0.6569626
\end{aligned}
$$




$$
\begin{aligned}
& \log \left[\mathrm{I}-\sqrt{\mathrm{I}-\frac{M^{2}}{\alpha^{2}}\left(\mathrm{I}-\alpha^{2}\right)}\right]=\overline{\mathrm{I}} .53534 \mathrm{I} 6 \\
& \mathrm{I}-\sqrt{\mathrm{I}-\frac{M^{2}}{\alpha^{2}}\left(\mathrm{I}-\alpha^{2}\right)}=0.3430374 \\
& \log \frac{\alpha}{M}=0.0735382 \\
& \text { sum }=\text { " } y_{\mathrm{m}}=\overline{\mathrm{I} .6088798}=\log \text { first approximation. }
\end{aligned}
$$

Carrying out the calculation by means of (33) we find $\log$ $q=\overline{2} .9887$ I $27, \log P=\overline{\mathrm{I}} .9271036$ (Table I) and, as a second approximation $\log y_{\mathrm{m}}=\overline{\mathrm{I}} .6088385$, which is a difference in the logarithm of $-4 \mathrm{I} 3$ in the seventh place. The factor $f$ for this case is 0.2874 (Table 3), which gives a final difference of -580 . Subtracting this from the logarithm of the first approximation, we have the final value $\log y_{\mathrm{m}}=\overline{\mathrm{r}} .60882 \mathrm{I} 8$. Using this value of $\log y_{m}$ in (33) the value found is $\log y_{m}=\overline{\mathrm{I}} .60882 \mathrm{I}$, which is sensibly the same, showing that the correct value is, in this case, found with only two applications of the main formula, and of these the last is necessary as a check only.

The value of the angle $\psi$ corresponding to the correct value of $y_{\mathrm{m}}$ is $46^{\circ} \mathrm{I} 3^{\prime} 22^{\prime \prime} .62$, which is only 0.2 of a minute less than the value taken from the curve. This corresponds to about I part in Io ooo difference between the correct value and the first approximation.

EXAMPLE 5.-FORMULA (37), USED IN CONNECTION WITH A FIRST APPROXIMATION TAKEN FROM THE CURVE (FIG. 2)

For $a=0.65$ the curve (Fig. 2) gives $\psi=46^{\circ} 28^{\prime} .5$, and a calculation exactly like that in the preceding example yields, as a first approximation, $\log y_{\mathrm{m}}=\overline{\mathrm{I}} .4750232$. Substituting this value in (37), we find $\log q_{1}=\overline{3} .6893759$, or $q_{1}=0.004890755$, to which corresponds $\log X=\overline{\mathrm{I}} .94267$ I I (Table 2 ), and the second approximation $\log y_{\mathrm{m}}=\overline{\mathrm{I}} \cdot 475055^{2}$. The logarithms of the first and second approximations here differ by 320 in the seventh place. For this case Table 3 gives $f=0.3725$, and the final correction is I.594 times 320 , or $5 \mathrm{rO}$, which, added to the first approximation, yields the value $\log y_{m}=\overline{\mathrm{r}} .4750742$. A check calculation using this value in (37) gives a value of the logarithm greater by one unit in the seventh place. The true value of $\psi$ is about 0.I 5 of a minute larger than the value taken from the curve. 
EXAMPLE 6.-CALCULATION OF THE FACTOR $P$ IN EQUATION (33a)

In the preceding examples the factor $P$ in equation (33a) has been obtained from Table I (see Appendix) by interpolation, using second and third differences. For this interpolation we use the formula

$$
P=P_{0}+h \Delta_{1}+\frac{h(h-\mathrm{I})}{L} \Delta_{2}+\frac{\dot{n}(h-\mathrm{I})(h-2)}{\underline{3}} \Delta_{3}+\ldots .
$$

where $P_{0}$ is the value of $P$ corresponding to the nearest tabular value $q_{0}$ of $q$, lower than that for which $P$ is desired, $h$ is the fraction of the tabular interval by which the actual value of $q$ exceeds $q_{0}$, and $\Delta_{1}, \Delta_{2}, \Delta_{3}$ are the first, second, and third differences, respectively, given in the table.

Assuming $q=0.1$ Ior 6235 , the value in example $\mathrm{r}$, we find

$$
\begin{aligned}
P_{0} & =0.808 \mathrm{I} 60 \quad \Delta_{1}=-3074.5 \quad \Delta_{2}=-\mathrm{I} 4 \\
h \Delta_{1} & =-499 \quad h=0.16235 \\
\frac{h(h-\mathrm{I})}{2} \Delta_{2} & =+\mathrm{I} \\
P & =\frac{.807662}{}
\end{aligned}
$$

In the relatively rare cases where the accuracy of the values of $P$, derived by interpolation from the Table $\mathrm{I}$, is not sufficient, we have to calculate the values of the terms of the series in (33) as was done in forming Table $\mathrm{I}$; retaining enough terms in the series and enough figures in the values of these terms to give the accuracy desired.

Thus for the value of $q$ above, we have $\log q=\overline{\mathrm{I}} .042033^{2}$, and the calculation of the terms may be arranged as below. In the first column are given the logarithms of the successive powers of $q$ required in (33). In the second column appear the logarithms of the terms of the series. These are obtained with little trouble if the logarithms of the constant coefficients in the series are written along the edge of a piece of paper, which may be placed under the numbers of the first column in such a way that the logarithm of any required coefficient may be brought directly under the logarithm of the corresponding power of $q$, and thus the addition of logarithms necessary may be readily carried out. In the third 
column are grouped the values of the terms of the series, the positive terms being grouped together first and under them the negative terms. The value of $P$ found shows that its value as interpolated from Table $I$ is accurate to the number of places given in the table.

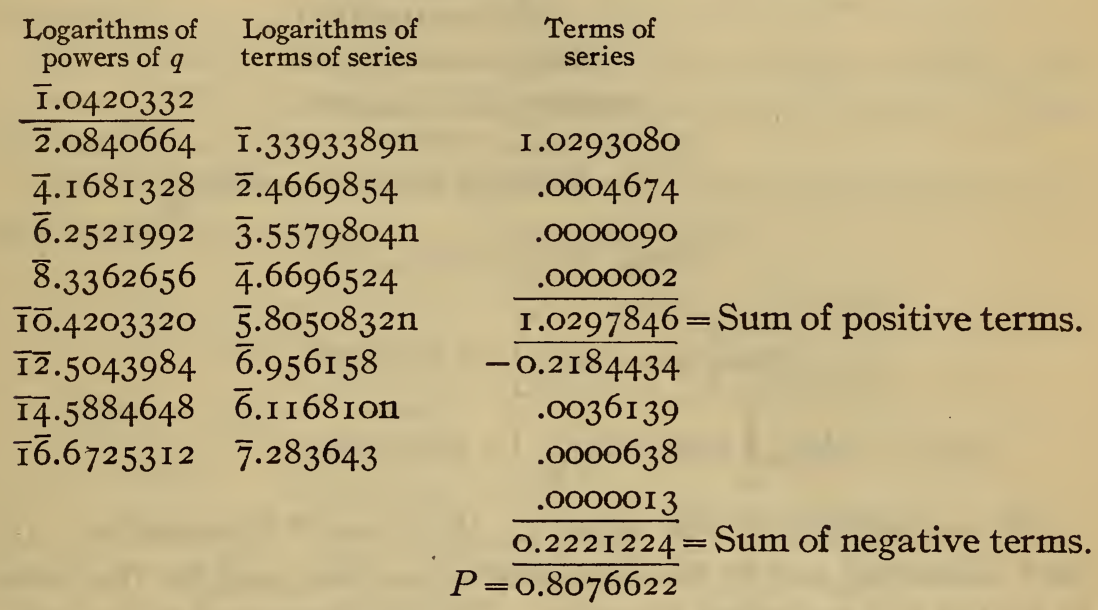

EXAMPLE 7.-CALCULATION OF THE FACTOR $X$ IIT EQUATIONS (37) AND (37a)

To illustrate the use of these formulas let us take the case assumed in example 3 , where we found $\log q_{1}=\overline{2} .0537527$.

The formula requires that the quantity $\log _{\ominus} \frac{\mathrm{I}}{q_{1}}$ shall be calculated. This may be quickly and accurately obtained by first writing $\log _{10} \frac{I}{q_{1}}$, and then performing the multiplication by the modulus $\left(\log _{e} \mathrm{IO}=2.302585 \mathrm{I}\right)$ by the aid of the special table provided for this purpose in collections of six-place and seven-place logarithms. The table referred to contains all the multiples of the modulus up roo times this quantity. By breaking up the desired logarithm into parts of two figures each the successive partial products may be written down directly from the table, the decimal point being properly placed in each case, and the sum of these products is the result desired. 
Thus in the present case

$$
\begin{aligned}
& \log _{10} q_{1}=\overline{2} .0537527 \\
& \log _{10} \frac{\mathrm{I}}{q_{1}}=\mathrm{I} .9462473
\end{aligned}
$$

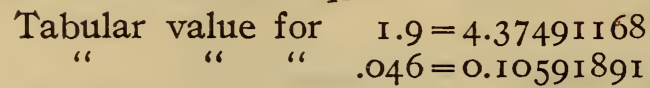

$$
\begin{aligned}
& .00024=0.00055^{262} \\
& .0000073=0.0000168 \mathrm{I} \\
& \mathrm{Sum}=4.48 \mathrm{I} 4000=\log _{\mathrm{e}} \frac{\mathrm{I}}{q_{1}} \\
& \log _{10} \log _{\mathrm{e}} \frac{\mathrm{I}}{q_{1}}=0.65 \mathrm{I} 4 \mathrm{I} 37 \\
& \log _{10}\left[\mathrm{I} 2 q_{1} \log _{\mathrm{e}} \frac{\mathrm{I}}{q_{1}}\right]=\mathrm{I} \cdot 7843476 \\
& \log _{10}\left[\mathrm{I} 20 q_{1}{ }^{2} \log _{\mathrm{e}} \frac{\mathrm{I}}{q_{1}}\right]=2.8381004
\end{aligned}
$$

The calculation of the series $Q, R, S$, and $T$ in equation (37) may be carried out by the same method as that used for the series in (33) in the preceding example. The logarithms of the ascending powers of $q_{1}$ enter into each of these series. Seven terms are necessary in order to obtain $Q$ to seven places, six terms for $R$, and only five each for $S$ and $T$.

Thus we find

$$
\begin{aligned}
& Q=0.8609019 \\
& R=0.837 \mathrm{I} 244 \\
& \text { I } 20 q_{1}^{2} R \log _{\mathrm{e}} \frac{\mathrm{I}}{q_{1}}=0.057662 \mathrm{I} \\
& C_{1}=0.8032398 \\
& S=\mathrm{I} .2767962 \\
& T=\mathrm{I} .025754^{2} \\
& \log C_{2}=\overline{\mathrm{r}} .8 \mathrm{I} 45803 \\
& \text { " } C_{1}=\overline{\mathrm{I}} .904845^{2} \\
& \text { “ } X=\overline{\mathrm{I}} .909735 \mathrm{I} \\
& X=0.8 \text { I } 23349
\end{aligned}
$$

The value of $X$ found by interpolation from Table 2, using the interpolation formula given in example 6 is $X=0.812335$. The amount of labor in a direct calculation of $X$ may be materially reduced if, in the computation of the smaller terms of the series, five-place logarithms are used. 
EXAMPLE 8.-USE OF EQUATIONS (38) AND (39)

Table 3, which is based on these equations, will suffice in the usual case, the required values of $f$ being found by interpolation. In cases where it may be necessary to calculate $f$ directly, the terms of the series in (38) may be obtained by the method of example 6.

For the case $\alpha=0.5$ the terms in (38) are I.87982, $0.0084 \mathrm{I}$, and 0.00020 , while the negative terms are 0.04420 , and 0.00125 , giving the result $f_{2}=0.92$ I $_{5}$, for the factor in (38).

For the use of (39) the results of the main calculation by (33) will usually be available. In the present case

$$
\begin{aligned}
& \log \text { Iо } q^{4}=\overline{3} \text {. I 74I } 28 \\
& \left(\mathrm{I}+\mathrm{rO} q^{4}\right)=\mathrm{I} .001493 \\
& \log \left(\mathrm{I}+\mathrm{IO} q^{4}\right)=\overline{0.000648} \\
& \log y_{\mathrm{m}}{ }^{2}=\overline{\mathrm{I}} . \mathrm{I} 67608 \\
& \text { " } k^{2}=\overline{\mathrm{I}} .92 \text { I } 344 \\
& \text { " }\left(\mathrm{I}+k^{\prime}\right)=0.148298 \\
& \mathrm{Sum}=\overline{\overline{\mathrm{I}} .237898} \\
& \text { I. } 7 \text { I } 5439 \\
& \log k^{\prime \prime}=\overline{\mathrm{I}} .609606 \\
& \frac{\mathrm{I}}{2} \text { “ } k^{\prime}=\overline{\mathrm{I}} .804803 \\
& \frac{3}{2} \quad \text { “ } k^{\prime}=\overline{\mathrm{I}} \cdot 4 \mathrm{I} 4409=\mathrm{Sum} \\
& \text { " } 4 \alpha=0.301030 \\
& \overline{\mathrm{I}} \text {.7 I } 5439=\mathrm{Sum} \\
& \text { Diff. }=\overline{\bar{I} \cdot 522459}=\log f_{1} \text {, equation (39) } \\
& \overline{\mathrm{I}} .96449 \mathrm{I}=\text { " factor of }(38) \\
& \operatorname{Sum}=\overline{\bar{I}} \cdot 486950=\log f \\
& 0.30687=f
\end{aligned}
$$

EXAMPLE 9.-CALCULATION BY FORMULAS (40), (41), AND (42)

Let us consider the same case as in the preceding example, $\alpha=0.5$.

$$
\begin{aligned}
& \log q_{1}=\overline{2} .053753 \\
& \log _{\mathrm{e}} \frac{\mathrm{I}}{q_{1}}=4.48 \text { I } 400 \text { (see example } 7 \text { ). }
\end{aligned}
$$

The calculation of the terms in the series for $\frac{d C_{1}}{d q_{1}}$ and $\frac{d C_{2}}{d q_{1}}$ in (40) offer nothing new, and will be found by the same method as in the preceding examples. The values found are $\frac{d C_{1}}{d q_{1}}=-17.2414$, and $\frac{d C_{2}}{d q_{1}}=-\mathrm{I} 9.443$. 
Making use of the values of $C_{1}$ and $C_{2}$ found in example 7 , the rest of the calculation of the factor $f$ follows readily.

$$
\begin{aligned}
& \frac{\mathrm{I}}{C_{2}} \frac{d C_{2}}{d q_{1}}=-29.798 \\
& \log k^{2}=\overline{\mathrm{I}} .92 \mathrm{I} 344 \\
& \text { " } \sqrt{k}=\overline{\mathrm{I}} .980336 \\
& \text { I } \frac{d C_{1}}{d q_{1}}=-2 \underline{1.465} \\
& \text { Diff. }=-8.333 \\
& \log \text { Diff. }=0.92080 \\
& q_{1}=\overline{2} .05375 \\
& \text { Sum }=\overline{\overline{2} .97455} \\
& -0.0943 \mathrm{I} \\
& \text { I.00000 } \\
& \mathrm{Sum}=0.90569 \\
& 1 / 2 “=0.45^{284} \\
& \text { factor in (40) } \\
& f=0.30677 \\
& \text { " } k^{\frac{5}{2}}=\overline{\mathrm{x}} \cdot 90168 \mathrm{o}=\mathrm{Sum} \\
& \text { “ } y_{\mathrm{m}}{ }^{2}=\overline{\mathrm{I}} \cdot \mathrm{I} 67608 \\
& \text { " }(\mathrm{I}+k)=0.28 \mathrm{I} 8 \mathrm{II} \\
& \text { Sum }=A=\overline{\mathrm{I}} \cdot 351099 \\
& \log _{4} \alpha=\overline{0.301030} \\
& \mathrm{Sum}=\mathrm{B}=\overline{\overline{\mathrm{I}} \cdot 52 \mathrm{O} 243} \\
& \text { Diff. }=(A-B)=\overline{\overline{\mathrm{I}} .830856}=\log \text { factor }(42) \\
& \log 0.45^{284}=\overline{\mathrm{I}} .655950 \\
& \log f=\overline{\overline{\mathrm{I}} .486806}
\end{aligned}
$$

EXAMPLE 10.-CALCULATION OF THE MAXIMUM FORCE BY FORMULAS (34) AND (5)

We have found in examples 2 and 3 for the case $\alpha=0.5$ the value of $\log y_{\mathrm{m}}=\overline{\mathrm{I}} .5838040$, to which corresponds $\log q=\overline{\mathrm{I}} .0435322$. This value of $q$ is the quantity which is designated by $q_{0}$ in equation (34).

In making the substitution of this value in (34) the method of example 6 gives for the terms in the parenthesis the following values:

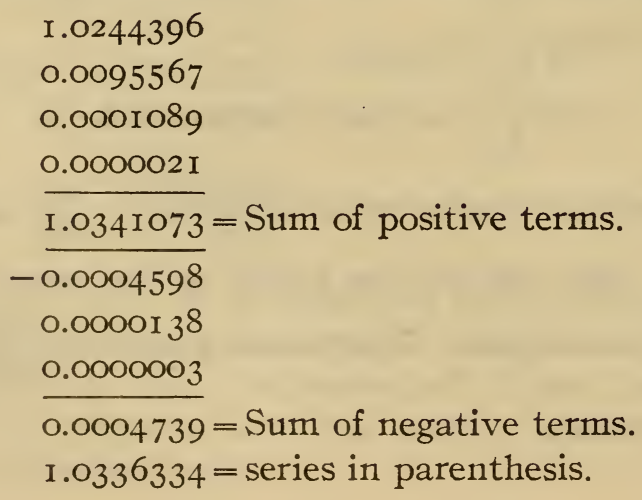


The remainder of the calculation is then

$$
\begin{aligned}
& \log \text { series }=0.0143666 \\
& \text { “ } \frac{96 \pi^{2}}{\sqrt{5}}=2.6270859 \\
& \text { “ } q_{0}^{2}=\overline{2.0870644} \\
& \text { “ } F_{\mathrm{m}}=\overline{0.7285169} \\
& F_{\mathrm{m}}=5.352010
\end{aligned}
$$

If, instead of using (34), we substitute the value of $y_{m}$ found previously in the general equation for the force, we should, of course, arrive at the same result, since equation (34) was derived by just this process. The value of $q$ will be the same as that in the preceding calculation. The terms in the series are found to be

$$
\begin{aligned}
& \text { I. } 2443963 \\
& 0.0335978 \\
& 0.0033575 \\
& 0.0002702 \\
& 0.0000185 \\
& 0.00000 \text { I I } \\
& \mathrm{Sum}=\overline{\mathrm{I.2816414}} \\
& \log \text { sum }=0.1077666 \\
& \text { “ } y_{m}=\overline{\mathrm{I}} \cdot 5^{8} 38040 \\
& \text { “ } \mathrm{I} 92 \pi^{2}=3.2776009 \\
& 2.969 \text { I } 7 \text { I } 5 \\
& \overline{3} \cdot 7593455 \\
& \log F_{\mathrm{m}}=0.7285 \mathrm{I} 7 \mathrm{O} \\
& \log q^{2}=\overline{2} .0870644 \\
& \text { " } \sqrt{q}=\overline{\mathrm{r}} .52 \mathrm{I} 766 \mathrm{I} \\
& \text { “ } q \frac{5}{2}=\overline{3} .6088305 \\
& \text { " } \sqrt{\alpha}=\underline{\mathrm{I}} .8494850 \\
& \overline{3} .7593455
\end{aligned}
$$

which gives a value of $F_{\mathrm{m}}$ in agreement with the result by the preceding calculation, thus checking (34).

\section{EXAMPLE 11.-CALCULATION OF THE MAXIMUM FORCE, FORMULAS} (37) AND (6)

The maximum force will be calculated in this example for the same case as in the preceding example to show the agreement of the formulas. In both cases the higher order terms of the series are important. The value of $q_{1}$, corresponding to the position of the coils for maximum force, has already been derived in example 3. It is $\log q_{1}=\overline{2} .05375^{27}$. 
The two sets of terms in the parentheses in (6) are then as follows:

$$
\begin{aligned}
& \text { I.I358107 I.0076852 } \\
& 0.0017854 \quad 0.0000213 \\
& \underline{0.0000040} \quad \underline{1.0077065} \text {, } \\
& \text { I.1 } 376006 \\
& -0.0245927-0.1131756 \\
& 0.0000924 \quad 0.0004349 \\
& 0.0000001 \quad 0.0000009 \\
& \text { Series }=\text { I.I I } 29154 \\
& -\overline{0.0246852}-\overline{0.1136114} \\
& \text { Second term }=0.544 \text { I } 659 \quad \text { I0.729I4I }=\text { multiplied by } 12 \text {. } \\
& \text { Parenthesis }=0.5687495 \\
& \log =\mathrm{I} .0305649 \\
& \log _{10} \log _{\ominus} \frac{\mathrm{I}}{q_{1}}=0.65 \mathrm{I} 4 \mathrm{I} 37 \\
& \log _{10} q_{1}=\underline{\overline{2} .0537527} \\
& \log \text { second term }=\overline{\mathrm{I}} .73573 \mathrm{I} 3 \\
& \log \text { parenthesis }=\overline{\mathrm{I}} .7549210 \\
& \log \frac{\pi}{16}=\overline{1} .2930299 \\
& \text { " } y_{\mathrm{m}}=\frac{\overline{\mathrm{I}} .5838040}{\overline{2} .6317549} \\
& \begin{aligned}
\log F_{m} & =\frac{\overline{3} .9032377}{0.7285 \text { I } 72} \\
F_{m} & =5.352014
\end{aligned} \\
& \begin{aligned}
\log _{6} \sqrt{\alpha} & =\overline{\mathrm{r}} .8494853 \\
q_{1} & =\frac{\overline{2} .05375^{27}}{\overline{3} .9032377}
\end{aligned}
\end{aligned}
$$

This result differs from that by the other independent formula (5) by no more than the errors of calculation with seven-place logarithms.

$$
\text { EXAMPLE 12.-THE COEFFICIENTS } \eta \text { AND } \epsilon
$$

These coefficients have been calculated for several values of $\alpha$ and are given in Table 5 . To show the agreement between the two sets of formulas, in the range where both may be used, the following values may be cited for the case $\alpha=0.5$.

$$
\begin{array}{llll} 
& \text { By (48) and (5I) } & \text { By (55) and (58) } & \begin{array}{l}
\text { B. S. current } \\
\text { balance }
\end{array} \\
\alpha=0.5 & \eta=-0.628 \mathrm{I} 3 & \eta=-0.62808 & \eta=-0.62 \\
& \epsilon=2.5494 & \epsilon=2.5493 & \epsilon=2.555
\end{array}
$$


For the case $\alpha=0.4$, the agreement of the two formulas is as good, the mean results being $\eta=-0.35298$ and $\epsilon=2.3102$. The values found experimentally with the $\mathrm{B}$. S. current balance were, for $\alpha=0.4, \eta=-0.35$ and $\epsilon=2.3 \mathrm{I} 7$. The agreement is to be regarded as satisfactory when it is remembered that the values calculated by the formulas are differential coefficients, whereas the experimental values refer to finite displacements.

\section{EXAMPLE 13.-ILLUSTRATING THE METHOD FOR CALCULATING THE MAXIMUM FORCE FOR COILS WHOSE RADII HAVE A RATIO DIFFERING SLIGHTLY FROM ONE OF THE VALUES OF TABLE 4}

Consider the case of a pair of coils whose radii were intended to have a ratio of $I: 2$, but whose actual values have a ratio of $\alpha=0.5$ (I.OI) $=0.505$.

Here $\frac{\Delta \alpha}{\alpha_{0}}=0.01$, if we take $\alpha_{0}=0.5$. For the value $\alpha_{0}=0.5$, Table 4 gives $\log F_{\mathrm{m}}=0.7285 \mathrm{I} 70$, and Table 5 gives for $\alpha=0.5$, $\epsilon=2.5493$; for $\alpha=0.55, \epsilon=2.7205$, with the tabular differences $\Delta_{1}=0.17$ I $2, \Delta_{2}=0.0507$, and $\Delta_{3}=0.0240$.

To obtain $\epsilon_{1}$, the value corresponding to $\alpha=0.505$, we have to interpolate for an interval of 0.1 of that used in the table, and we find the result $\epsilon_{1}=2.5493+0.017 \mathrm{I}-0.0023=2.564 \mathrm{I}$.

This gives, therefore, as a first approximation, $\frac{\Delta F}{F_{0}}=0.01 \times 2.56$ and, consequently, $\frac{F_{1} \alpha_{0}}{F_{0} \alpha_{1}}=\frac{\mathrm{I} .0256}{\mathrm{I} . \mathrm{OI}}=\mathrm{I} .0 \mathrm{I} 545$, and $\epsilon_{1}{ }^{\prime}=2.564 \mathrm{I}$ $X 1.01545=2.6037$. As a first approximation, therefore, the mean value over the interval is $\frac{\mathrm{I}}{2}(2.5493+2.6037)=2.5765$. Using this value, we have, as a second approximation, $\frac{F_{1} \alpha_{0}}{F_{0} \alpha_{1}}=\frac{\text { I.025765 }}{\mathrm{I} . \mathrm{OI}}$ $=\mathrm{I} .0156 \mathrm{I}$, which gives $\epsilon_{1}{ }^{\prime}=2.564 \mathrm{I} \times \mathrm{I} .0156 \mathrm{I}=2.604 \mathrm{I}$, and the second approximation to the mean value is $\epsilon=2.5767$, which is very little different from the first approximation.

We have, therefore, $\frac{\Delta F}{F_{0}}=0.025767$, and the required value of $F_{\mathrm{m}}$ for $\alpha=0.505$ is found by multiplying the tabular value of $F_{\mathrm{m}}$ for $\alpha_{0}=0.5$ by the factor 1.025767 . 


$$
\begin{aligned}
& \log (\mathrm{I} .025767)=0.0110487 \\
& \text { " } F_{0}=0.7285170 \\
& \log F_{\mathrm{m}}=0.7395657 \quad F_{\mathrm{m}}=5.4899 \mathrm{I} 6 \text { for } \alpha=0.505 \text {. }
\end{aligned}
$$

To check this calculation, we may find the value of $\eta$ for this same interval. Table 5 gives

$$
\begin{aligned}
\eta_{0} & =-0.628 \text { Io for } \alpha_{0}=0.5 \\
\eta_{1} & =-0.6459 \mathrm{I} \text { interpolated for } \alpha_{1}=0.505 \\
\eta_{1} \frac{\alpha_{0}}{\alpha_{1}} \frac{y_{1}}{y_{0}} & =-0.6459 \mathrm{I}\left(\frac{\mathrm{I}-.00636}{\mathrm{I} .0 \mathrm{I}}\right)=-0.63545 \\
\eta_{1}{ }^{\prime} & =-0.63 \mathrm{I} 78
\end{aligned}
$$

A second approximation gives $\eta_{1}{ }^{\prime}=-0.63$ I 79. Using the value $\frac{\Delta y}{y_{0}}=-0.0063179$, we find $\overline{\mathrm{I}} \cdot 5^{8105 \mathrm{I} 4}$ as the value of $\log y_{\mathrm{m}}$ derived from the tabular value for $\alpha_{0}=0.5$. Using this as a first approximation, we find by use of the main equation (33) the slightly more accurate value $\log y_{\mathrm{m}}=\overline{\mathrm{I}} .5^{8105 \mathrm{I} 7}$ for $\alpha=0.505$, and substituting this result in (34) the force is found to have a value of 5.489926 , a value only two parts in a million different from the value found by the interpolation process. This difference is not large when we consider the largeness of the interval over which the interpolation was made and the very considerable magnitude of the second and third differences of the tabulated values of $\epsilon$. The amount of work necessary for finding the value of the maximum force by use of the interpolated value of $\epsilon$ is, of course, vastly less than that required for the application of the exact formulas.

EXAMPLE 14.-CALCULATION OF THE MAXIMUM FORCE BETWEEN THE BUREAU OF STANDARDS CURRENT BALANCE COILS AND THE DISTANCES BETWEEN THE COILS

To illustrate the use of the formulas of this article for actual coils, the complete calculations are given of the constants of the coils of the Bureau of Standards current balance. The work is arranged for easier following in tabular form (Tables A and B); all the steps, except some of minor importance, are given in the compass of the table. For comparison, the published results given in Tables IX and XI of the article on the determination of 
the international ampere are appended. The latter values, as has already been explained, were obtained by interpolation between the values of the force calculated by formula (4) for a number of distances of the coils in the neighborhood of the position corresponding to maximum force.

\section{TABLE A}

Calculation of Maximum Distance $z_{\mathrm{m}}$ for Bureau of Standards Coils

\begin{tabular}{|c|c|c|c|c|c|c|c|c|}
\hline Coils & $\frac{\Delta \alpha}{\Delta}$ & $\begin{array}{l}\text { Interpo- } \\
\text { lated } \\
\text { value } \eta_{1}\end{array}$ & $=\eta_{1} \frac{\eta_{1}^{\prime}}{\alpha_{0}} \frac{y_{\mathrm{m}}}{\alpha_{1}} \frac{y_{0}}{y_{0}}$ & ${ }_{\text {mean }}^{\eta}$ & $\frac{\Delta y}{y_{0}}$ & $\log y_{m}$ & $z_{\mathrm{m}}$ & $\begin{array}{l}\text { Values } \\
\text { current } \\
\text { balance }\end{array}$ \\
\hline $\mathrm{M}_{2} \mathrm{I}_{11}$ & -0.0010800 & -0.62620 & -0.62715 & -0.62755 & +.0006778 & $\overline{1} .5840983$ & 9.60946 & 9.6092 \\
\hline $\mathrm{M}_{2} \mathrm{I}_{2}$ & -.0010566 & .62625 & .62733 & .62772 & 6632 & .5840919 & 9.60910 & 9.6089 \\
\hline $\mathrm{M}_{2} \mathrm{I}_{3}$ & +.0002793 & .62859 & .62831 & .62822 & -.0001755 & .5837278 & 9.58822 & 9.5882 \\
\hline $\mathrm{M}_{2} \mathrm{I}_{4}$ & .0000908 & .62826 & .62817 & .62813 & - $\quad 570$ & .5837792 & 9.59117 & 9.5912 \\
\hline $\mathrm{M}_{3} \mathrm{~S}_{1}$ & +.0047609 & -0.63652 & -0.63158 & -0.62984 & -.0029986 & $\overline{1} .5824998$ & 7.64009 & 7.6400 \\
\hline $\mathrm{M}_{3} \mathrm{~S}_{2}$ & .0052139 & .63731 & .63190 & .63000 & 32848 & .5823750 & 7.63445 & 7.6344 \\
\hline $\mathrm{M}_{3} \mathrm{~L}_{1}$ & +.0022365 & -0.35492 & -0.35384 & -0.35341 & -.0007904 & $\overline{1} .6294549$ & 10.66733 & 10.6673 \\
\hline $\mathrm{M}_{3} \mathrm{~L}_{2}$ & .0022600 & .35503 & .35394 & .35346 & 7981 & .6294515 & 10.66700 & 10.6669 \\
\hline $\mathrm{M}_{3} \mathrm{~L}_{3}$ & .0036000 & .35611 & .35438 & .35368 & -.0012732 & .6292449 & 10.64769 & 10.6476 \\
\hline $\mathrm{M}_{3} \mathrm{~L}_{4}$ & .0034113 & .35595 & .35431 & .35364 & 12064 & .6292741 & 10.65041 & 10.6502 \\
\hline $\mathrm{M}_{4} \mathrm{~L}_{1}$ & -.0038325 & -0.62133 & -0.62520 & -0.62665 & +.0024016 & $\overline{1} .5848458$ & 9.62602 & 9.6256 \\
\hline $\mathrm{M}_{4} \mathrm{~L}_{2}$ & -.0038091 & .62127 & .62512 & .62661 & 23868 & .5848394 & 9.62565 & 9.6253 \\
\hline $\mathrm{M}_{4} \mathrm{I}_{3}$ & -.0024768 & .62365 & .62617 & .62714 & 15533 & .5844781 & 9.60480 & 9.6047 \\
\hline $\mathrm{M}_{4} \mathrm{~L}_{4}$ & -.0026649 & .62341 & .62611 & .62711 & 16712 & .5845292 & 9.60775 & 9.6077 \\
\hline
\end{tabular}

It must be remembered that the maximum force here calculated is not quite equal to the maximum force between the actual coils, but is the value between the equivalent filaments by which the coils may be replaced, on the assumption that their cross sections are square. It is the force $F_{1}$ in the article referred to, and the correction for the actual deviation from a square cross section must be applied as there shown (pp. 329 to 334 ).

The equivalent radii of the coils, derived by Lyle's Method, are given there in Table IX. They are:

\begin{tabular}{|c|c|c|c|}
\hline Coil & $\begin{array}{c}\text { Equivalent } \\
\text { radius }\end{array}$ & Coil & $\begin{array}{c}\text { Equivalent } \\
\text { radius }\end{array}$ \\
\hline $\mathbf{M}_{2}$ & 12.50552 & $\mathbf{S}_{2}$ & 19.97113 \\
$\mathbf{M}_{3}$ & 10.03763 & $\mathbf{L}_{1}$ & 25.03808 \\
$\mathbf{M}_{4}$ & 12.47106 & $\mathbf{L}_{2}$ & 25.03749 \\
$\mathbf{S}_{1}$ & 19.98014 & $\mathbf{L}_{3}$ & 25.00405 \\
& & $\mathbf{L}_{4}$ & 25.00877 \\
\hline
\end{tabular}


Table A covers the calculation of the distance $z_{\mathrm{m}}$ for maximum force. Column 2 gives the fractional differences between the actual ratios of the radii and the nominal values $\alpha_{0}$. The latter is $\alpha_{0}=0.4$ for the movable coil $M_{3}$, when used with the fixed coils $L_{1}, L_{2}, L_{3}$, and $L_{4}$ : for the other combinations it is 0.5 . Column 3 gives the interpolated values of $\eta_{1}$, and column 4 the same quantity multiplied by the correction factor $\frac{\alpha_{0}}{\alpha_{1}} \frac{y_{1}}{y_{0}}$.

In columns 5 and 6 appears the mean value $\eta$ and the change in $y_{0}, \frac{\Delta y}{y_{0}}=\eta \frac{\Delta \alpha}{\alpha_{0}}$ for the actual fractional deviations of the coils from the nominal ratio of the radii. The values of column 7 are found by adding the logarithms of $\left(x+\frac{\Delta y}{y_{0}}\right)$ to the values of $\log$ $y_{0}$ for the nominal ratio taken from Table 4 , viz:

For $\alpha_{0}=0.5 \log y_{0}=\overline{\mathrm{I}} .5838040$
" $\alpha_{0}=0.4$ " $y_{0}=\overline{\mathrm{I}} .6297983$

TABLE B

Calculation of the Maximum Force for the Bureau of Standards Coils

\begin{tabular}{|c|c|c|c|c|c|c|c|c|c|}
\hline Coils & $\frac{\Delta \alpha}{\alpha_{0}}$ & $\begin{array}{c}\text { Inter- } \\
\text { polated } \\
\text { value } \\
\varepsilon_{1}\end{array}$ & $=\varepsilon_{1} \frac{{\stackrel{\alpha_{0}}{\prime}}_{\alpha_{1}}^{\prime} F_{m}}{\alpha_{1} F_{0}}$ & mén & $\frac{\Delta F}{F_{0}}$ & $\log \left(\frac{1+\Delta F}{F_{0}}\right)$ & $\log F_{1}$ & $F_{1}$ & $\begin{array}{c}F_{1} \\
\text { values } \\
\text { current } \\
\text { balance }\end{array}$ \\
\hline $\mathbf{M}_{2} \mathrm{~L}_{3}$ & +0.0002793 & 2.5493 & 2.5510 & 2.5502 & +0.0007123 & 0.0003092 & 0.7288262 & 5.355823 & 5.355834 \\
\hline $\mathbf{M}_{2} \mathrm{~L}_{4}$ & +.0000908 & 2.5493 & 2.5499 & 2.5496 & .0002315 & .0001006 & 0.7286176 & 5.353251 & 5.353251 \\
\hline $\mathbf{M}_{3} \mathbf{S}_{1}$ & +0.0047609 & 2.5563 & 2.5754 & 2.5623 & +0.0121987 & 0.0052658 & 0.7337828 & 5.417298 & 5.417298 \\
\hline $\mathrm{M}_{3} \mathrm{~S}_{2}$ & .0052139 & 2.5569 & 2.5776 & 2.5635 & .0133658 & .0057662 & 0.7342832 & 5.423544 & 5.423552 \\
\hline $\mathbf{M}_{3} \mathbf{L}_{1}$ & +0.0022365 & 2.3118 & 2.3186 & 2.3144 & +0.0051761 & 0.0022422 & 0.4966225 & 3.137780 & 3.137781 \\
\hline $\mathbf{M}_{3} \mathbf{L}_{2}$ & .0022600 & 2.3119 & 2.3187 & 2.3145 & .0052308 & .0022658 & .4966461 & 3.137951 & 3.137953 \\
\hline $\mathbf{M}_{3} \mathrm{~L}_{3}$ & .0036000 & 2.3130 & 2.3239 & 2.3171 & .0083416 & .0036077 & .4979880 & 3.147662 & 3.147665 \\
\hline $\mathbf{M}_{3} \mathrm{~L}_{4}$ & .0034113 & 2.3129 & 2.3232 & 2.3167 & .0079030 & .0034187 & .4977990 & 3.146292 & 3.146292 \\
\hline$M_{4} L_{3}$ & -0.0024768 & 2.5458 & 2.5360 & 2.5426 & -0.0062974 & $\overline{1} .9972564$ & 0.7257734 & 5.318308 & 5.318310 \\
\hline $\mathrm{M}_{4} \mathrm{~L}_{4}$ & -.0026649 & 2.5454 & 2.5349 & 2.5421 & -.0067744 & 9970479 & .7255649 & 5.315754 & 5.315754 \\
\hline
\end{tabular}

The values of the distance $z_{\mathrm{m}}$ then follow on multiplying the values in column 7 by the radius $A$ of the larger coil. The values published in Table IX appear in the last column. The differences between the latter and the values here calculated are very small and are not systematic. 
In Table B appear the calculations of the maximum force. These should be clear from the description of the preceding table. The values of the maximum force $F_{0}$, corresponding to the nominal ratios 0.5 and 0.4 , are taken from Table 4 , and form the basis of the calculations. In the last column are given the values of $F_{\mathrm{m}}$ from Table XI of the article on the current balance. The differences between these values and those here calculated are, on the average, less than a part in a million. In fact, the agreement is better than for the distances $z_{\mathrm{m}}$ in the preceding table. This is explained when it is remembered that the force changes very slowly in the neighborhood of the critical distance, and thus makes it difficult to obtain the actual position of the coil for maximum force by interpolation.

\section{SUMMARY}

I. The force of attraction or repulsion between two parallel, coaxial circular currents has its maximum value for a distance $z_{\mathrm{m}}$, between their planes, such that the ratio $\frac{z_{m}}{A}$ is a function of the ratio of the radii $\frac{a}{A}$ alone.

2. The value of the force between the two circular filaments, when one ampere flows in each, is a function of the ratio of the radii alone. A knowledge of the maximum value of this force is of practical importance in the absolute measurement of current, since the Rayleigh current balance employs two coils placed experimentally at the distance which gives the maximum force between them. The calculation of the constant of this current balance postulates a knowledge of the maximum value of the force in terms of the dimensions of the coils.

4. Formulas have been developed by Maxwell and Nagaoka which allow the force to be calculated between any two parallel, coaxial currents whose planes are at any desired distance $z$ apart. Previously the maximum value of the force has been derived by interpolation, graphically or otherwise, between values of the force calculated for various positions of the coils in the region where the force is known to be a maximum. 
5. In this paper, by a method which is an extension of that used by Nagaoka in deriving his formulas for the force between the coils, two equations are derived, which give the distance between the coils corresponding to maximum force, as a function of the ratio of the radii, $\alpha$. These relations are in the form of $q$ series, one depending on a modulus which is complementary to that of the other. One or the other of these formulas will be found to converge well for any desired value of $\alpha$.

6. Unfortunately, these relations do not, apparently, allow of being put in a form such as to allow the distance $z_{\mathrm{m}}$ to be calculated directly. They may, however, be solved for $z_{\mathrm{m}}$ by successive approximation without serious difficulty.

7. Starting from Rayleigh's approximation formula for $z_{\mathrm{m}}$, which gives precise values over only a limited range of values of the ratio of the radii, methods are here worked out for facilitating the process of approximation, together with an empirical method for obtaining a more accurate first approximation than can, in general, be obtained by the formula of Rayleigh.

8. Tables are given of the distance $z_{\mathrm{m}}$ and the maximum force $F_{\mathrm{m}}$ for values of the ratio of the radii advancing in steps of 0.05 , as well as a number of tables of auxiliary quantities, which materially reduce the labor of calculation of the distance $z_{\mathrm{m}}$ and the maximum force for such values of $\alpha$ as are not included in the tables.

9. A method is also given for easily obtaining the values of the desired quantities from the fundamental values in the tables for coils having radii whose ratio differs only slightly from one of the nominal values included in the tables. This covers the case of current balance coils in practice.

Io. The use of the formulas and tables is thoroughly illustrated by examples so designed as to show the agreement of the different methods. Finally, a comparison is given of the results of calculations made for the coils of the Bureau of Standards current balance, by the method of this paper, with the published values obtained by the interpolation process. The agreement is, on the average, closer than one part in a million. For practical cases like this the use of the tables given here enables the constants to 
be found with vastly less labor than in a general case not covered by the fundamental values in the tables. Even in the general case, however, the method of this paper is likely to be less time consuming than the interpolation method, and has the advantage of furnishing a check on the latter.

WASHINGTON, January I, I9I 5. 


\section{APPENDIX}

TABLE 1

Table of Values of the Quantity in Brackets in Equation 33 (Factor P in Equation (33a))

\begin{tabular}{|c|c|c|c|c|c|c|c|}
\hline$q$ & $P$ & $\Delta_{1}$ & $\Delta_{2}$ & $q$ & $P$ & $\Delta_{1}$ & $\Delta_{2}$ \\
\hline 0 & 1.000000 & -18 & -36 & 0.040 & 0.971701 & -1406 & -32.5 \\
\hline 0.001 & 0.999982 & 54 & 36 & .041 & .970295 & 1438.5 & 32 \\
\hline .002 & .999928 & 90 & 36 & .042 & .968856 & 1470.5 & 31.5 \\
\hline .003 & .999838 & 126 & 36 & .043 & .967386 & 1502 & 32 \\
\hline .004 & 999712 & 162 & 36 & .044 & .9658835 & 1534 & 31 \\
\hline .005 & 999550 & 198 & 36 & .045 & .964350 & 1565 & 31 \\
\hline 0.006 & 0.999352 & -234 & -36 & 0.046 . & 0.962784 & -1596 & -31.5 \\
\hline .007 & 9991185 & 270 & 35.5 & .047 & .961188 & 1627.5 & 31 \\
\hline .008 & .998849. & 305.5 & 35.5 & .048 & 959560 & 1658 & 31 \\
\hline .009 & 998543 & 341 & 36 & .049 & .957902 & 1689 & 30 \\
\hline .010 & .998202 & 377 & 36 & .050 & .956213 & 1719 & 30 \\
\hline 0.011 & 0.997825 & -413 & -35 & 0.051 & 0.954494 & -1749.5 & -30 \\
\hline .012 & .997412 & 448 & 36 & .052 & .952744 & 1779.5 & 30 \\
\hline .013 & 996964 & 484 & 36 & .053 & .950965 & 1809 & 29.5 \\
\hline .014 & 996480 & 520 & 35 & .054 & 9491555 & 1839 & 29 \\
\hline .015 & 995960 & 555 & 35 & .055 & .947317 & 1868 & 29 \\
\hline 0.016 & 0.995405 & -590 & -36 & 0.056 & 0.945449 & -1897 & -29 \\
\hline .017 & .994815 & 626 & 35 & .057 & 943552 & 1926 & 29 \\
\hline .018 & 994189. & 661 & 35 & .058 & 941626 & 1954.5 & 28.5 \\
\hline .019 & 993528 & 696 & 35 & .059 & .939671 & 1983 & 28 \\
\hline .020 & .992832 & 731 & 35 & .060 & .937688 & 2011 & 28 \\
\hline 0.021 & 0.992101 & -766 & -35 & 0.061 & 0.935677 & -2039 & -28 \\
\hline .022 & .991334 & 801 & 35 & $\begin{array}{r}.062 \\
\end{array}$ & .933638 & 2067 & 27 \\
\hline .023 & 990533. & 836 & 34 & .063 & .9315715 & 2094 & 27 \\
\hline .024 & 989698 & 870 & 35 & .064 & .929478 & 2121 & 27 \\
\hline .025 & .988827 & 905 & 34 & .065 & .927356 & 2148 & 27 \\
\hline 0.026 & 0.987922 & -939 & -35 & 0.066 & 0.925208 & -2175 & -26 \\
\hline .027 & 986983 & 974 & 34 & .067 & . .9230335 & 2201 & 26 \\
\hline .028 & 986009 & 1007 & 34 & .068 & .920832 & 2227 & 26 \\
\hline .029 & .985002 & 1042 & 34 & .069 & .918605 & 2253 & 26 \\
\hline .030 & .983960 & 1076 & -33.5 & .070 & .916352 & 2279 & 25 \\
\hline 0.031 & 0.982884 & -1109.5 & -33.5 & 0.071 & 0.914073 & -2304 & -25 \\
\hline .032 & 9817745 & 1143 & 34 & .072 & .911769 & 2329 & 25 \\
\hline .033 & .980631 & 1177 & 33 & .073 & .909440 & 2354 & 24 \\
\hline .034 & 979455 & 1210 & 33 & .074 & .9070855 & 2379 & 24 \\
\hline .035 & .978245 & 1243 & 33 & .075 & .904707 & 2403 & 24 \\
\hline 0.036 & 0.977002 & -1276 & -33 & 0.076 & 0.902304 & -2427 & -24 \\
\hline .037 & .975726 & 1309 & 32.5 & .077 & .899877 & 2451 & 23.5 \\
\hline .038 & 974417 & 1341.5 & 32.5 & .078 & .897426 & 2474 & 23 \\
\hline .039 & .973075 & 1374 & 32 & .079 & .894952 & 2497 & 23 \\
\hline .040 & .971701 & 1406 & 32.5 & .080 & .892455 & 2520 & 23 \\
\hline
\end{tabular}


TABLE 1-Continued

Table of Values of the Quantity in Brackets in Equation 33 (Factor P in Equation (33a))-Continued

\begin{tabular}{|c|c|c|c|c|c|c|c|}
\hline$q$ & $P$ & $\Delta 1$ & $\Delta^{2}$ & $q$ & $P$ & $A$ & $\Delta^{2}$ \\
\hline 0.080 & 0.892455 & -2520 & -23 & 0.100 & 0.838072 & -2919 & -17 \\
\hline .081 & .889934 & 2543 & 22.5 & .101 & .835153 & 2935 & 17 \\
\hline .082 & .8873915 & 2565 & 22 & .102 & .8322175 & 2952 & 16 \\
\hline .083 & .884826 & 2587 & 22 & .103 & .829265 & 2968 & 16 \\
\hline .084 & .882239 & 2609 & 22 & .104 & .826297 & 2985 & 15 \\
\hline .085 & .879630 & 2631 & 21.5 & .105 & .823312 & 3000 & 15 \\
\hline 0.086 & 0.876999 & -2652 & -21 & 0.106 & 0.820312 & -3015.5 & -15 \\
\hline .087 & .874347 & 2673 & 21 & .107 & .8172965 & 3031 & 15 \\
\hline .088 & .871674 & 2694 & 20.5 & .108 & .814266 & 3046 & 14 \\
\hline .089 & .868981 & 2713.5 & 20 & .109 & .811220 & 3060 & 14 \\
\hline .090 & .866267 & 2734 & 19.5 & .110 & .808160 & 3074.5 & 14 \\
\hline 0.091 & 0.863533 & -2754 & -19 & 0.111 & 0.805086 & -3088 & -14 \\
\hline .092 & .860779 & 2773 & 19.5 & $: 112$ & .801998 & 3102 & 13.5 \\
\hline .093 & .858006 & 2793 & 19 & .113 & .798896 & 3115 & 13 \\
\hline .094 & .855213 & 2811 & 19 & .114 & .795781 & 3128 & 13 \\
\hline .095 & .852402 & 2830 & 18 & .115 & .792652 & 3141 & 13 \\
\hline 0.096 & 0.849572 & -2848 & -18 & 0.116 & 0.789511 & -3154 & -12 \\
\hline .097 & .846724 & 2867 & 17.5 & .117 & .786358 & 3166 & 12 \\
\hline .098 & .843857 & 2884 & 17.5 & .118 & .783192 & 3178 & 12 \\
\hline .099 & .840973 & 2901.5 & 17 & .119 & .780014 & 3190 & 12 \\
\hline .100 & .838072 & 2919 & 17 & .120 & .776825 & 3202 & 12 \\
\hline
\end{tabular}

TABLE 2.

Table of Values of Factor $X$ in Equation (37'a.)

\begin{tabular}{|c|c|c|c|c|c|c|c|}
\hline $\mathrm{q}_{1}$ & $x$ & $\Delta_{1}$ & $\Delta_{2}$ & $q_{1}$ & $x$ & $\Delta_{1}$ & $\Delta_{2}$ \\
\hline 0 & 1.000000 & -7058 & +1654 & 0.0015 & 0.942127 & -2667 & +69.5 \\
\hline 0.0001 & 0.992942 & 5404 & 620 & .0016 & .939461 & 2597 & 65.5 \\
\hline .0002 & .9875375 & 4784 & 400 & .0017 & .9368635 & 2532 & 62 \\
\hline .0003 & .982753 & 4384 & 296 & .0018 & .934332 & 2470 & 58 \\
\hline .0004 & .978369 & 4088 & 235 & .0019 & .931862 & 2412 & 54 \\
\hline .0005 & .974281 & 3853 & 195 & .0020 & .929450 & 2357 & 52 \\
\hline 0.0006 & 0.970428 & -3658 & +166 & 0.0021 & 0.927093 & -2305 & +50 \\
\hline .0007 & .966770 & 3493 & 144 & .0022 & .9247875 & 2256 & 47 \\
\hline .0008 & .963277 & 3348 & 128 & .0023 & .922532 & 2208 & 45 \\
\hline .0009 & 959929 & 3221 & 114 & .0024 & .920324 & 2163 & 43 \\
\hline .0010 & .956708 & 3107 & 104 & .0025 & .918160 & 2120 & 41 \\
\hline 0.0011 & 0.953601 & -3002.5 & +94 & 0.0026 & 0.916040 & -2079 & +39 \\
\hline .0012 & .950599 & 2909 & 87 & .0027 & .913962 & 2039 & 38 \\
\hline .0013 & .947690 & 2822 & 80.5 & .0028 & 911922 & 2001 & 37 \\
\hline .0014 & 944869 & 2741 & 74.5 & .0029 & 909921 & 1964 & . 35 \\
\hline .0015 & .942127 & 2667 & 69.5 & .0030 & 907957 & 1929 & 34 \\
\hline
\end{tabular}


TABLE 2-Continued

Table of Values of Factor $X$ in Equation (37'a.) - Continued

\begin{tabular}{|c|c|c|c|c|c|c|c|}
\hline$q_{1}$ & $\mathrm{x}$ & $S_{1}$ & $\Delta_{2}$ & $\mathrm{q}_{1}$ & $\mathrm{x}$ & $\Delta_{1}$ & $\Delta_{2}$ \\
\hline 0.0030 & 0.907957 & -1929 & +34 & 0.0075 & 0.844435 & -1029 & +11 \\
\hline .0031 & .906028 & 1895 & 33 & .0076 & .843406 & 1018 & 12 \\
\hline .0032 & .904133 & 1862 & 32 & .0077 & .842388 & 1006 & 12 \\
\hline .0033 & .902271 & 1830.5 & 31 & .0078 & .8413825 & 994 & 11 \\
\hline .0034 & .900440 & 1800 & 30 & .0079 & .8403885 & 983 & 12 \\
\hline .0035 & .898640 & 1770 & 29 & .0080 & .839406 & 971 & 11 \\
\hline 0.0036 & 0.896870 & -1741 & +28 & 0.0081 & 0.838435 & -960 & +11 \\
\hline .0037 & .895129 & 1713 & 27 & .0082 & .837474 & 949 & 11 \\
\hline .0038 & .893416 & 1686 & 26 & .0083 & .836525 & 938 & 10 \\
\hline .0039 & .891730 & 1660 & 25 & .0084 & .835587 & 928 & 10.5 \\
\hline .0040 & .890070 & 1634 & 25 & .0085 & .834659 & 917.5 & 10.5 \\
\hline 0.0041 & 0.888436 & -1610 & +25 & 0.0086 & 0.833742 & -907 & +10 \\
\hline .0042 & .886826 & 1585 & 24 & .0087 & .832835 & 897 & 10 \\
\hline .0043 & .885241 & 1561.5 & 23. & .0088 & .831938 & 887 & 10 \\
\hline .0044 & .883679 & 1539 & 22 & .0089 & .831051 & 877 & 10.5 \\
\hline .0045 & .882141 & 1516.5 & 22 & .0090 & .830174 & 867.5 & 9.5 \\
\hline 0.0046 & 0.880624 & -1495 & +21 & 0.0091 & 0.829307 & -858 & +10 \\
\hline .0047 & .8791295 & 1473.5 & 21 & .0092 & .828449 & 848 & 9 \\
\hline .0048 & .877656 & 1453 & 21 & .0093 & .827601 & 839 & 10 \\
\hline .0049 & .876203 & 1432 & 19 & .0094 & .826763 & 829 & 8 \\
\hline .0050 & .874771 & 1413 & 19.5 & .0095 & .825933 & 821 & 10 \\
\hline 0.0051 & 0.873359 & -1393 & +19 & 0.0096 & 0.825113 & -811 & +8 \\
\hline .0052 & .871965 & 1374 & 18 & .0097 & .8243015 & 803 & 10 \\
\hline .0053 & .870591 & 1356 & 18 & .0098 & 823499 & 793 & 8 \\
\hline .0054 & .869235 & 1338 & 17.5 & .0099 & .822705 & 785 & 9 \\
\hline .0055 & .867898 & 1320 & 17 & .0100 & .821920 & 776 & 8 \\
\hline 0.0056 & 0.8665775 & -1303 & +17 & 0.0101 & 0.821144 & -768 & +8 \\
\hline .0057 & .865275 & 1286 & 16.5 & .0102 & .820375 & 760 & 8 \\
\hline .0058 & .863989 & 1269 & 16 & .0103 & .819616 & 752 & 9 \\
\hline .0059 & .862720 & 1253 & 16 & .0104 & .818864 & 743 & 7 \\
\hline .0060 & .861467 & 1236.5 & 15.5 & .0105 & .818121 & 736 & 8 \\
\hline 0.0061 & 0.8602305 & -1221 & +15 & 0.0106 & 0.817385 & -728 & +8 \\
\hline .0062 & .859009 & 1206 & 15 & .0107 & .816657 & 720 & 8 \\
\hline .0063 & .857803 & 1191 & 15 & .0108 & .815938 & 712 & 7 \\
\hline .0064 & .856613 & 1176 & 14.5 & .0109 & .815226 & 705 & 9 \\
\hline .0065 & .8554365 & 1161 & 14 & .0110 & .814521 & 696 & $?$ \\
\hline 0.0066 & 0.8542755 & -1147 & +13.5 & 0.0111 & 0.813825 & -689 & +7 \\
\hline .0067 & .853128 & 1133.5 & 13 & .0112 & .813136 & 682 & 7 \\
\hline .0068 & .851995 & 1119 & 13 & .0113 & .812454 & 675 & 8 \\
\hline .0069 & .850876 & 1106 & 13 & .0114 & .811779 & 667 & 7 \\
\hline .0070 & .849770 & 1093 & 13 & .0115 & .811112 & 660 & 6.5 \\
\hline 0.0071 & 0.848677 & -1080 & +13 & 0.0116 & 0.810452 & -653.5 & +7.5 \\
\hline .0072 & .847597 & 1066 & 13 & .0117 & .8097985 & 646 & 7 \\
\hline .0073 & .846531 & 1054 & 12 & .0118 & .809153 & 639 & 6.5 \\
\hline . 0074 & .845477 & 1042 & 13 & .0119 & .8085135 & 632.5 & 6.5 \\
\hline .0075 & .844435 & 1029 & 11 & .0120 & .807881 & 626 & 6 \\
\hline
\end{tabular}


TABLE 3

Values of Factors in Differential Equations (38) to (42)

\begin{tabular}{|c|c|c|c|c|c|c|c|c|}
\hline \multirow{2}{*}{$\alpha=\frac{a}{A}$} & \multicolumn{2}{|c|}{ logarithms of factors } & \multirow{2}{*}{$\log f$} & \multirow{2}{*}{$f$} & \multirow{2}{*}{$\begin{aligned} & \log F \\
= & \log \frac{I}{I-f}\end{aligned}$} & \multirow{2}{*}{$F$} & \multirow{2}{*}{$\Delta_{1}$} & \multirow{2}{*}{$\Delta_{2}$} \\
\hline & in (38) & in (39) & & & & & & \\
\hline 0 & $1.69897 \mathrm{n}$ & $\overline{1} .60206 \mathrm{n}$ & $\overline{1} .30103$ & 0.20000 & 0.09691 & 1.2500 & +18 & +34 \\
\hline 0.05 & .70209 & .60136 & .30345 & .20112 & .09752 & .2518 & 52 & 37 \\
\hline 0.10 & .71137 & .59926 & .31063 & .20447 & .09934 & .2570 & 89 & 36 \\
\hline 0.15 & .72657 & .59573 & .32231 & .21004 & .10240 & .2659 & 125 & 39 \\
\hline 0.20 & .74735 & .59072 & .33807 & .21780 & .10668 & .2784 & 164 & 41 \\
\hline 0.25 & .77328 & .58406 & .35734 & .22769 & .11221 & .2948 & 205 & 42 \\
\hline 0.30 & $\overline{1} .80383$ & $\overline{1} .57587$ & $\overline{1} .37969$ & 0.23971 & 0.11902 & 1.3153 & +247 & +46 \\
\hline 0.35 & .83860 & .56579 & .40438 & .25374 & .12711 & .3400 & 293 & 48 \\
\hline 0.40 & $.87716^{\circ}$ & .55370 & .43086 & .26969 & .13649 & .3693 & 341 & 52 \\
\hline 0.45 & 91919 & .53936 & .45855 & .28744 & .14718 & .4034 & 393 & 55 \\
\hline 0.50 & $\overline{1} .96448$ & .52246 & .48695 & .30686 & .15918 & .4427 & 448 & 58 \\
\hline 0.55 & 0.01292 & $\overline{1} .50259$ & .51551 & .32773 & 0.17246 & 1.4875 & 506 & \\
\hline \multirow[b]{2}{*}{$\alpha$} & \multicolumn{2}{|c|}{ logarithms of factors } & \multirow{2}{*}{$\log f$} & \multirow[b]{2}{*}{$f$} & \multirow[b]{2}{*}{$\log F$} & \multirow[b]{2}{*}{$F$} & \multirow[b]{2}{*}{$\Delta_{1}$} & \multirow[b]{2}{*}{$\Delta g$} \\
\hline & $\ln 40$ & in 42 & & & & & & \\
\hline 0.45 & $\overline{1} .65906$ & $\overline{1} .79941$ & $\overline{1} .45847$ & 0.28739 & 0.14715 & 1.4033 & +392 & +55 \\
\hline 0.50 & .65595 & .83086 & .48681 & .30677 & .15912 & .4425 & 447 & 56 \\
\hline 0.55 & 1.65597 & 1.85936 & .51534 & 0.32759 & 0.17237 & 1.4872 & +503 & +58 \\
\hline 0.60 & .65834 & .88523 & .54357 & .34960 & .18682 & .5375 & 561 & 55 \\
\hline 0.65 & .66246 & 90864 & .57110 & .37248 & .20237 & .5936 & 616 & 47 \\
\hline 0.70 & .66782 & 92970 & .59755 & .39584 & .21885 & .6552 & 663 & 30 \\
\hline 0.75 & .67391 & 94841 & .62232 & .41910 & .23590 & .7215 & 693 & +1 \\
\hline 0.80 & $\overline{1} .68030$ & 1.96471 & $\overline{1} .64501$ & 0.44159 & 0.25305 & 1.7908 & 694 & -50 \\
\hline 0.85 & .68659 & .97845 & .66504 & .46241 & .26955 & .8602 & 644 & -132 \\
\hline 0.90 & .69229 & .98932 & .68161 & .48041 & .28434 & .9246 & 512 & -270 \\
\hline 0.95 & .69678 & $\overline{1} .99684$ & .69362 & .49388 & .29575 & 1.9758 & 242 & \\
\hline 1.00 & $\overline{1} .69897$ & 0.00000 & $\overline{1} .69897$ & 0.50000 & 0.30103 & 2.0000 & & \\
\hline
\end{tabular}




\section{TABLE 4}

Fundamental Values of the Maximum Force Ratio $y_{\mathrm{m}}=\frac{z_{\mathrm{m}}}{A}$ and the Maximum Force $F_{\mathrm{m}}$

\begin{tabular}{|c|c|c|c|c|c|c|c|}
\hline$\alpha=\frac{a}{A}$ & $\log y_{m}$ & $y_{m}$ & \multicolumn{3}{|c|}{$\psi$} & $\log F_{\mathrm{m}}$ & $F_{\mathrm{m}}$ \\
\hline 0 & $\overline{1} .6989700$ & 0.5000000 & $\begin{array}{c}0 \\
45\end{array}$ & '́ & $\begin{array}{c}\prime \prime \prime \\
11.8\end{array}$ & & 0 \\
\hline 0.05 & .6979913 & .4988745 & 45 & 50 & 31.04 & 2.6279557 & 0.04245763 \\
\hline 0.10 & .6950381 & .4954936 & 45 & 51 & 28.52 & $\overline{1} .2326395$ & 0.1708596 \\
\hline 0.15 & .6900572 & .4898434 & 45 & 53 & 3.47 & $\overline{1} .5892443$ & 0.3883688 \\
\hline 0.20 & .6829567 & .4818998 & 45 & 55 & 14.40 & $\overline{1} .8454176$ & 0.7005153 \\
\hline 0.25 & .6736010 & .4716296 & 45 & 57 & 59.40 & 0.0475214 & 1.1156332 \\
\hline 0.30 & $\overline{1} .6618033$ & 0.4589901 & 46 & 01 & 15.60 & 0.2163112 & 1.6455504 \\
\hline 0.35 & .6473125 & .4439279 & 46 & 04 & 58.77 & 0.3629849 & 2.306667 \\
\hline 0.40 & .6297983 & .4263815 & 46 & 09 & 3.72 & 0.4943803 & 3.121622 \\
\hline 0.45 & .6088217 & .4062765 & 46 & 13 & 22.62 & 0.6150973 & 4.121898 \\
\hline 0.50 & .5838040 & .3835341 & 46 & 17 & 45.84 & 0.7285170 & 5.352011 \\
\hline 0.55 & $\overline{1} .5539627$ & 0.3580657 & 46 & 21 & 59.60 & 0.8373702 & 6.876544 \\
\hline 0.60 & .5182254 & .3297808 & 46 & 25 & 45.82 & 0.9441070 & 8.792392 \\
\hline 0.65 & .4750743 & .2985893 & 46 & 28 & 39.62 & 1.0512140 & 11.25159 \\
\hline 0.70 & .4222883 & .2644163 & 46 & 30 & 8.59 & 1.1615723 & 14.50682 \\
\hline 0.75 & .3564223 & .2272073 & 46 & 29 & 26.32 & 1.2790169 & 19.01152 \\
\hline 0.80 & $\overline{1} .2717509$ & 0.1869610 & 46 & 25 & 30.92 & 1.4094251 & 25.66995 \\
\hline 0.85 & $\overline{1} .1576668$ & .1437695 & 46 & 17 & 1.94 & 1.5633218 & 36.58658 \\
\hline 0.90 & $\overline{2} .9905951$ & .0978577 & 46 & 02 & 2.59 & 1.7638911 & 58.06188 \\
\hline 0.95 & $\overline{2} .6960993$ & .0496706 & 45 & 37 & 59.40 & 2.0847542 & 121.54978 \\
\hline 1.00 & & 0 & 45 & 0 & 0.00 & & \\
\hline
\end{tabular}




\section{TABLE 5}

Coefficients $\eta$ and $\epsilon$ in Equations (48), (51), (55), and (58)

\begin{tabular}{|c|c|c|c|c|c|c|c|c|}
\hline$\alpha=\frac{a}{A}$ & $\eta$ & $\Delta_{1}$ & $\Delta_{2}$ & $\Delta_{3}$ & $\varepsilon$ & $\Delta_{1}$ & $\Delta_{2}$ & $\Delta_{3}$ \\
\hline 0 & 0 & -.00451 & -.00919 & -.00050 & 2.0000 & +.0040 & +.0082 & +.0003 \\
\hline 0.05 & -0.00451 & 1370 & 969 & 87 & .0040 & 122 & 85 & 8 \\
\hline 0.10 & .01821 & 2339 & 1056 & 129 & .0162 & 207 & 93 & 12 \\
\hline 0.15 & .04160 & 3395 & 1185 & 193 & .0369 & 300 & 105 & 14 \\
\hline 0.20 & .07555 & 4580 & 1378 & 263 & .0669 & 405 & 119 & 22 \\
\hline 0.25 & .12135 & 5958 & .01641 & 366 & 2.1074 & 524 & 141 & 33 \\
\hline 0.30 & -0.18093 & -.07599 & -.02007 & -.00516 & 2.1598 & +.0665 & +.0174 & +.0043 \\
\hline 0.35 & .25692 & .09606 & 2523 & 731 & .2263 & 839 & 217 & 62 \\
\hline 0.40 & .35298 & .12129 & 3254 & 1083 & .3102 & .1056 & 279 & 98 \\
\hline 0.45 & .47427 & .15383 & 4337 & -.01573 & .4158 & .1335 & 377 & 130 \\
\hline 0.50 & .62810 & .19720 & -.0591 & -.0262 & 2.5493 & .1712 & 507 & 237 \\
\hline 0.55 & -0.82530 & -.2563 & -.0853 & -.0395 & 2.7205 & +.2219 & +.0744 & +.0380 \\
\hline 0.60 & 1.0816 & .3416 & .1248 & .0763 & 2.9424 & .2963 & .1124 & 690 \\
\hline 0.65 & 1.4232 & .4664 & .2011 & .1466 & 3.2387 & .4087 & . 1814 & .1206 \\
\hline 0.70 & 1.8896 & .6675 & .3477 & .3391 & 3.6474 & .5901 & .3020 & .3790 \\
\hline 0.75 & 2.5571 & 1.0152 & .6868 & -1.0101 & 4.2375 & .8921 & .6810 & 9679 \\
\hline 0.80 & -3.5723 & -1.7020 & -1.6969 & -5.0143 & 5.1496 & +1.5731 & +1.6489 & +4.9548 \\
\hline 0.85 & 5.2743 & 3.3989 & 6.7112 & & 6.7127 & 3.2220 & 6.6037 & \\
\hline 0.90 & 8.6722 & 10.1101 & & & 9.9347 & 9.8257 & & \\
\hline 0.95 & -18.7823 & & & & 19.7604 & & & \\
\hline
\end{tabular}

